Louisiana State University

LSU Digital Commons

$1-1-2018$

\title{
Design of SECAR a recoil mass separator for astrophysical capture reactions with radioactive beams
}

\author{
G. P.A. Berg \\ University of Notre Dame \\ M. Couder \\ University of Notre Dame \\ M. T. Moran \\ University of Notre Dame \\ K. Smith \\ University of Notre Dame \\ M. Wiescher \\ University of Notre Dame
}

See next page for additional authors

Follow this and additional works at: https://digitalcommons.Isu.edu/physics_astronomy_pubs

\section{Recommended Citation}

Berg, G., Couder, M., Moran, M., Smith, K., Wiescher, M., Schatz, H., Hager, U., Wrede, C., Montes, F., Perdikakis, G., Wu, X., Zeller, A., Smith, M., Bardayan, D., Chipps, K., Pain, S., Blackmon, J., Greife, U., Rehm, K., \& Janssens, R. (2018). Design of SECAR a recoil mass separator for astrophysical capture reactions with radioactive beams. Nuclear Instruments and Methods in Physics Research, Section A: Accelerators, Spectrometers, Detectors and Associated Equipment, 877, 87-103. https://doi.org/10.1016/ j.nima.2017.08.048

This Article is brought to you for free and open access by the Department of Physics \& Astronomy at LSU Digital Commons. It has been accepted for inclusion in Faculty Publications by an authorized administrator of LSU Digital Commons. For more information, please contact ir@lsu.edu. 


\section{Authors}

G. P.A. Berg, M. Couder, M. T. Moran, K. Smith, M. Wiescher, H. Schatz, U. Hager, C. Wrede, F. Montes, G.

Perdikakis, X. Wu, A. Zeller, M. S. Smith, D. W. Bardayan, K. A. Chipps, S. D. Pain, J. Blackmon, U. Greife, K. E. Rehm, and R. V.F. Janssens 


\title{
Design of SECAR a Recoil Mass Separator for Astrophysical Capture Reactions with Radioactive Beams
}

\author{
G.P.A. Berg ${ }^{1}$, M. Couder, M.T. Moran, K. Smith ${ }^{2}$, \\ M. Wiescher \\ Department of Physics and The Joint Institute for Nuclear Astrophysics, \\ University of Notre Dame, Notre Dame, Indiana 46556, USA
}

$$
\text { H. Schatz, F. Montes, G. Perdikakis, }
$$

National Superconducting Cyclotron Laboratory and The Joint Institute for Nuclear Astrophysics, Michigan State University, East Lansing, Michigan 48824, USA

$$
\text { U. Hager, X. Wu }{ }^{4} \text {, A. Zeller }
$$

National Superconducting Cyclotron Laboratory, Michigan State University, East Lansing, Michigan 48824, USA

$$
\text { C. Wrede }
$$

Department of Physics and Astronomy and the National Superconducting Cyclotron Laboratory, Michigan State University, East Lansing, MI 48824, USA

$$
\text { M.S. Smith, D.W. Bardayan }{ }^{3} \text {, K.A. Chipps, S.D. Pain }
$$

Oak Ridge National Laboratory, USA

\author{
J. Blackmon \\ Louisiana State University, USA \\ U. Greife
}

Department of Physics, Colorado School of Mines, Golden, Colorado 80401, USA

K.E. Rehm, R.V.F. Janssens

Argonne National Laboratory, USA 


\begin{abstract}
A recoil mass separator SECAR has been designed for the purpose of studying low-energy $(p, \gamma)$ and $(\alpha, \gamma)$ reactions in inverse kinematics with radioactive beams for masses up to about $\mathrm{A}=65$. The reaction rates are of importance for our understanding of the energy production and nucleosynthesis during explosive hydrogen and helium burning. The radiative capture reactions take place in a windowless hydrogen or He gas target at the entrance of the separator, which consists of four Sections. The first Section selects the most abundant charge state. The second and third Sections contain Wien Filters providing high mass resolving power to separate efficiently the intense beam from the few reaction products. In the following fourth Section, the reaction products are guided into a detector system capable of positon, angle and time-of-flight measurements. In order to accept the complete kinematic cone of recoil particles including multiple scattering in the target in the center of mass energy range of $0.2 \mathrm{MeV}$ to $3.0 \mathrm{MeV}$, the system must have a large polar angle acceptance of $\pm 25 \mathrm{mrad}$. This requires a careful minimization of higher order aberrations. The present system will be installed at the NSCL ReA3 accelerator and will be used with the much higher beam intensities of the FRIB facility when it becomes available.
\end{abstract}

PACS: 07.55.-w; 07.55.+h; 29.30.Aj; 41.85.-p; 41.75.-i; 25.40.Lw; 26.50.+x:

Keywords: Recoil mass separator, Wien Filter, Radiative proton and alpha capture

\title{
1 Introduction
}

A large number of radiative captures rates of proton and alpha particles on unstable proton-rich nuclei need to be determined with much better precision than presently possible to interpret observations and address open questions related to nova explosions [1] and X-ray bursts [2] that occur in the dense and hot atmospheres on the surfaces of accreting white dwarfs and neutron stars, respectively. At sufficiently high temperatures and densities on the surface of neutron stars, a sequence of $(\mathrm{p}, \gamma)$ reactions and $\beta$-decays, known as the rpprocess, follows the breakout from the hot CNO cycles and was first described by Wallace and Woosley [3] to explain the explosions in these cataclysmic binary star systems.

Since the $(\mathrm{p}, \gamma)$ reactions cannot be measured directly on short-lived unstable target nuclei, several pioneering recoil separators have used inverse kinematics

1 Corresponding author, Fax: +1-574-631-5952, E-mail: gpberg@bergs.com

2 Present address: University of Tennessee, Knoxville, USA

3 Present address: University of Notre Dame, Notre Dame, IN 46556,USA

4 Present address: Ionetix Corporation, Lansing, MI 48911, USA 
and radioactive beams to measure a few stellar reaction rates, mainly in the mass region below $\mathrm{A}=30$. Examples are the DRS separator at the HRIBF facility in Oak Ridge [4], the DRAGON separator at TRIUMF [5] and the ARES separator at Louvain-la-Neuve [6]. A recent overview of the experimental technique and existing recoil systems can be found in [7].

The measurement of low energy reactions using stable beams in inverse kinematics was pioneered by Kremer at al. [8] who applied this technique to the reaction ${ }^{12} \mathrm{C}(\alpha, \gamma){ }^{16} \mathrm{O}$ and showed that coincidences between $\gamma$ radiation and recoil particles provide a very efficient tool for reducing background originating from beam, environment and cosmic radiation.

Subsequently, several recoil mass separators have been designed and built using this technique for stable nuclei. Examples are the ERNA separator [9] at the DTL laboratory in Bochum and the St. GEORGE separator [10] at the University of Notre Dame. The very high beam suppression allows measurements at energies lower than possible with the traditional $\gamma$-detection method. In these direct $(p, \gamma)$ experiments, the nuclear reaction cross sections are measured by detecting $\gamma$ rays emitted from the reaction products. Since the cross sections drop exponentially toward stellar energies, measurements of the $\gamma$ radiation to determine the stellar reaction cross sections are limited by natural radiogenic and cosmogenic as well as the beam-related background radiation. Therefore, stellar reaction rates, that depend on the resonant and possibly non-resonant reaction contribution cannot be determined by the $\gamma$-detection method [11].

Present separators at radioactive beam facilities are limited to a few favorable reactions with sufficient luminosities. With the advent of high-intensity radioactive beam facilities like RIKEN, FAIR and FRIB, sufficiently high luminosities are available for a larger number of reactions to be studied. This calls for a recoil separator that allows systematic measurements on protonrich nuclei in the range of target masses of $\mathrm{A}=20$ - 65 appropriate for the rp-process.

For inverse kinematics reactions of radiative capture, the reaction products are emitted in a narrow cone at forward angles because the $\gamma$ particle does not carry much momentum. For the same reason, the momentum of the beam and reaction products are nearly identical and a magnetic analysis alone does not provide sufficient separation. At the low beam energies considered here, the simple "wedge method" $[12,13]$ that is being used for mass separation at higher energies is not applicable. Therefore, a recoil mass separator applying magnetic and electric field must provide the separation based on the mass difference of the beam and the reaction products.

On the basis of known principles and other recoil separators, in particular 
the recoil separator St. GEORGE, that is optimized for low energy $(\alpha, \gamma)$ reactions with stable beam nuclei, the recoil SEparator for CApture Reactions SECAR has been designed for low energy $(p, \gamma)$ reactions with intense radioactive beams in inverse kinematics for masses up to about $\mathrm{A}=65$.

SECAR has been designed, like the St. GEORGE recoil separator, with Wien filters (WF) with crossed magnetic and electric fields, allowing for a very compact design. In a Wien filter, also known as "velocity filter" particles with a velocity $v=E / B$ travel straight through the system, while particles with different velocities are deflected. In the case, where the momentum $p=m \cdot v$ is fixed for a given beam energy, the Wien filter acts as a "mass filter".

The first-order "mass resolving power" is defined as $\mathrm{R}_{m}=\left(\mathrm{x} \mid \delta_{m}\right) /\left((\mathrm{x} \mid \mathrm{x}) \cdot 2 \mathrm{x}_{0}\right)$, where $\left(\mathrm{x} \mid \delta_{m}\right)$ is the mass dispersion, $(\mathrm{x} \mid \mathrm{x})$ the magnification and $2 \mathrm{x}_{0}$ the full target (object) spot size. The mass resolving power is defined in analogy to the well-known momentum resolving power $\left(\mathrm{x} \mid \delta_{p}\right) /\left((\mathrm{x} \mid \mathrm{x}) \cdot 2 \mathrm{x}_{0}\right)$ where $\left(\mathrm{x} \mid \delta_{p}\right)$ is the momentum dispersion. The "mass resolving power" of the recoil separator shown here are $\approx \mathrm{R}_{m}=740$ at FP2 and 1300 at FP3. The "mass resolution" $\mathrm{R}_{H O}$ is defined in the same way as $\mathrm{R}_{m}$, except that the first-order image size $(\mathrm{x} \mid \mathrm{x}) \cdot 2 \mathrm{x}_{0}$ is replaced by the real object size that can be calculated by including the higher order $(\mathrm{HO})$ aberrations. A resolution as large as the resolving power can only be achieved, if contributions of $\mathrm{HO}$ aberrations to the first order image size $\left.(\mathrm{x} \mid \mathrm{x}) \cdot 2 \mathrm{x}_{0}\right)$ are negligible.

For the $(\mathrm{p}, \gamma)$ reaction on target masses up to about $\mathrm{A}=65$ that are of interest for the rp-process, the relative mass difference can be as small as $\Delta \mathrm{m} / \mathrm{m}=$ $1 / 65$, or - in the notation we will be using - the mass separation has to be larger than $\mathrm{m} / \Delta \mathrm{m}=65$. Because of the overwhelmingly large beam intensity compared to the extremely small rates of the reaction products, however, the mass resolution has to be much larger to provide a beam rejection that allows a measurement of the reaction products above the beam tail. The mass resolving power of a separator can be defined with good precision. However, since it is impossible to predict the beam distribution precisely at a large distance from the beam center, the beam rejection cannot be predicted reliably. Therefore, we define the Nominal Beam Rejection NBR(D) by assuming a Gaussian spatial distribution for beam and reaction products with the same $\sigma$. The NBR(D) is defined as the integral of the tail of a standard normal Gaussian distribution $\phi(\mathrm{x})$ at a distance $\mathrm{D}$ from the center of the distribution

$$
N B R(D)=\int_{D-2 \sigma}^{D+2 \sigma} \phi(x) \mathrm{d} x
$$

This integral represents the ratio of about $95 \%$ of the counts of the reaction products in an interval of length $\pm 2 \sigma$ and the counts of the beam tail in the same interval where the integral over the beam is normaized to 1 . 
While this definition of the Nominal Beam Rejection is a useful tool to optimize a design and to compare different recoil separators it is not a realistic method to determine the experimental beam rejection, because a real beam is not expected to be represented by a Gaussian distribution at a distance from the beam center. In addition, other background sources from beam scattering and charge exchange on the residual gas and scattering from chamber walls may be the limiting factor of the beam rejection. We have, therefore, used the following reasoning for the design mass separation. DRAGON is designed for masses up to about $\mathrm{A}=30$ and demonstrates a beam rejection of about $10^{-13}$ for a mass resolution of $\mathrm{R}_{H O} \approx 350$. For a similar beam rejection but for masses up to $\mathrm{A}$ $=65$ we require a mass resolution of a factor of 2 larger, namely $\mathrm{R}_{H O}>700$, if all other conditions like vacuum and acceptances are equal.

Additional, demanding requirements are essential for a successful design of a recoil separator.

(1) The presence of several charge states created in the target at low energies demands a physical charge selection.

(2) With decreasing energy multiple scattering effects increase and require angle acceptances larger than the kinematic cone of the reactions and, therefore, large magnets with large gaps and Good-Field-Regions (GFR).

(3) The large acceptances make a careful correction of higher order (HO) aberrations necessary.

(4) While the detector system is able to help identify particles at the end of the recoil separator, it is important to reduce the background as much as possible using halo rejecting slits to stay within the rate capability of the detectors.

These in part conflicting requirements must be considered and incorporated in an optimized recoil separator suitable for the intended astrophysical studies. In the following section we will describe general design considerations followed by the description of the layout of the recoil separator. Then, we will discuss the ion optics, the design of the magnets and Wien filters. Before concluding the article with a summary, we will present an alternate version of the recoil separator with only one Wien filter.

\section{Design considerations}

Low-energy radioactive beam facilities such as the CRC/Louvain la Neuve (LLN), HRIBF/ORNL, and ISAC/TRIUMF have identified the goals and the technical requirements for studying astrophysical capture reactions in inverse kinematics. All these facilities identified a recoil mass separator as one of the most important tools for the measurement of low-energy reactions with 
rare isotope beams near the astrophysically relevant energy range, and consequently installed such a device to carry out their pioneering measurements.

The beam rejection and range of possible reactions goals for SECAR significantly exceed those in existing recoil separators. The Daresbury Recoil Separator, DRS, now installed at ORNL's HRIBF was not designed specifically for capture reactions and was only later optimized for such an application. It was very recently used for a measurement of the ${ }^{17} \mathrm{~F}(\mathrm{p}, \gamma){ }^{18} \mathrm{Ne}$ reaction [20] and typically has beam rejections of $10^{-8}$ to $10^{-10}$. ARES was a low-budget separator designed from existing parts at Louvain La Neuve for an inverse kinematics measurement of a single reaction, namely the ${ }^{19} \mathrm{Ne}(\mathrm{p}, \gamma){ }^{20} \mathrm{Ne}$ reaction. DRAGON was the first dedicated recoil separator designed, built, and optimized to study capture reactions for nuclear astrophysics with rare isotope beams. The first reaction with unstable beam measure with DRAGON was the ${ }^{21} \mathrm{Na}(\mathrm{p}, \gamma)^{22} \mathrm{Mg}$ reaction [21]. It is a very successful device that has enabled a number of pioneering nuclear astrophysics measurements and will continue to be a productive facility. It achieves a beam rejection ranging from $10^{-8}$ to $10^{-13}$ and typically in the $10^{-9}-10^{-10}$ range for lower energy measurements of astrophysical interest [15] and is optimized for reactions with beams of mass numbers $A<30$. In addition, there are other separator devices that have not been specifically used for radioactive beam measurements of capture reactions, but have been used as recoil separators and, therefore, provide useful insights such as the FMA at ANL, ERNA in Bochum,that now has been moved to Caserta, Italy to be utilized in a modified version adding a dipole magnet to the system and St.GEORGE at Notre Dame.

Based on the extensive experience with these separators, ARES (CRC) [6], DRS (HRIBF) [14], and DRAGON (TRIUMF) [15] five critical performance requirements were identified:

(1) Large acceptance angle for full transmission of recoil products.

(2) Unique charge state identification and separation of recoil particles.

(3) Primary beam rejection with a maximum of leaky beam rates of about $10^{-2}$ to $10^{-3}$ pps at the focal plane of the separator.

(4) Design for a broad range of capture reactions, not just a few benchmarks.

(5) Target and focal plane detector systems that provide about $10^{-4}$ additional rejection of the primary beam.

These requirements form the basis of the technical design of SECAR. Assuming beam intensities at FRIB of up to $10^{11} \mathrm{pps}$, leaky beam rates of about $10^{-2}-10^{-3}$ pps require a rejection of $10^{-13}-10^{-14}$, which is significantly better than existing devices, where beam rejection is the fraction of the beam passing through a slit set as narrow as possible but still transmitting nearly all reaction products. 
A leaky beam rate of the order of $10^{-2}$ pps is sufficiently high to allow monitoring the performance of the separator during the experiment, and is sufficiently low so that the detector system can provide the remaining rejection required to carry out the experiment. The high rejection capability must be achieved for a wide range of reactions, with proton captures on heavier isotopes posing a particular challenge because at larger masses the relative mass difference between beam and recoil becomes smaller.

Because absolute cross sections need to be determined, it is essential that the recoil separator transmits all recoils of the selected charge state. Kinematic calculations were performed to establish relevant parameters for the design, in particular the required angular acceptances and rigidities. For the reliable setup and tuning of the device it is desirable to have enough bending power to also transmit the primary beam without having to change the incident beam energy.

Table 1 shows the results of the calculations for a range of typical proton capture reactions, together with ${ }^{15} \mathrm{O}(\alpha, \gamma)$ and ${ }^{44} \mathrm{Ti}(\alpha, \gamma)$, and the resulting requirements for rigidities and angular acceptances. We show results for two extreme values of center of mass beam energies $\mathrm{E}_{C M}$ and for a range of capture reactions that are representative for the energies, $\mathrm{E}_{C M}$, to be investigated at NSCL and FRIB.

As Table 1 shows, maximum magnetic and electric rigidities of $0.8 \mathrm{Tm}$ and 19 MV are sufficient for all recoils and most of the beams, except for measurements at $3 \mathrm{MeV}$ center of mass energy for targets with mass numbers $\mathrm{A}>30$ where magnetic rigidities $>0.8 \mathrm{Tm}$ are needed. However, the table displays the most extreme range possible. In practice, measurements will typically be performed below $1 \mathrm{MeV}$ center of mass energies. For these energies the rigidities are small enough so that the beam can be transmitted. The recoil energy spread is relatively small and ranges from $0.1 \%$ to $2.3 \%$ for $(\mathrm{p}, \gamma)$ rates and up to $3.1 \%$ for ${ }^{15} \mathrm{O}(\alpha, \gamma)$.

Table 1 also shows, that a maximum angular acceptance of $\pm 25 \mathrm{mrad}$ is required for ${ }^{15} \mathrm{O}(\alpha, \gamma)$ with the largest scattering angle of $15.6 \mathrm{mrad}$ of the listed examples because beam spot effects broaden the angular range, and because it is not desirable to be close to the walls defining the acceptance. This acceptance is more than sufficient for all proton capture reactions. The SECAR acceptance of $\pm 25 \mathrm{mrad}$ for $100 \%$ transmission is larger than the measured acceptance of $\pm 17 \mathrm{mrad}$ of the DRAGON separator.

In summary, the general requirements for SECAR are a beam rejection of $10^{-13}-10^{-14}$ just from the separator for beam masses up to about $\mathrm{A}=65$, and additional rejection of $10^{-4}$ from auxiliary detectors, a maximum magnetic rigidity of $0.8 \mathrm{Tm}$, a maximum elecric rigidity of $19 \mathrm{MV}$, an angular acceptance 
of $\pm 25 \mathrm{mrad}$, and an energy acceptance of $\pm 3.1 \%$. These design parameters are summarized in Table 2.

\section{$3 \quad$ SECAR design and layout}

The layout of the separator in the existing ReA3 Experimental Hall at NSCL, MSU is shown in Fig. 1. The SECAR system starts with JENSA [22] a high density windowless gas jet target TGT. JENSA is already installed at the

Table 1

Reactions of astrophysical interest together with kinematic paramaters that determine the design parameters.

\begin{tabular}{|c|c|c|c|c|c|c|c|c|}
\hline & & \multicolumn{7}{|c|}{ Inverse reaction } \\
\hline Reaction & $\mathrm{E}_{c m}$ & Q-value & dE/E & Recoil & Half & B $\rho$ & E $\rho$ & B $\rho$ \\
& Beam & {$[19]$} & Range & Charge & Angle & Recoil & Recoil & Beam \\
& $\mathrm{MeV}$ & $\mathrm{MeV}$ & $\pm \%$ & $\mathrm{q}$ & mrad & Tm & MV & Tm \\
\hline${ }^{15} \mathrm{O}(\alpha, \gamma){ }^{19} \mathrm{Ne}$ & 0.5 & 3.528 & 3.1 & 3 & 15.6 & 0.29 & 1.25 & 0.14 \\
& 3.0 & 3.528 & 2.1 & 6 & 10.3 & 0.35 & 3.75 & 0.35 \\
${ }^{44} \mathrm{Ti}(\alpha, \gamma){ }^{48} \mathrm{Cr}$ & 0.5 & 7.698 & 2.3 & 4 & 11.7 & 0.58 & 2.74 & 0.19 \\
& 3.0 & 7.698 & 1.3 & 10 & 6.2 & 0.57 & 6.59 & 0.48 \\
${ }^{19} \mathrm{Ne}(p, \gamma){ }^{20} \mathrm{Na}$ & 0.2 & 2.190 & 1.3 & 4 & 6.4 & 0.31 & 1.88 & 0.21 \\
& 3.0 & 2.190 & 0.71 & 9 & 3.6 & 0.54 & 12.5 & 0.81 \\
${ }^{23} \mathrm{Mg}(p, \gamma)^{24} \mathrm{Al}$ & 0.2 & 1.863 & 0.91 & 4 & 4.6 & 0.38 & 2.28 & 0.15 \\
& 3.0 & 1.863 & 0.56 & 11 & 2.8 & 0.53 & 12.4 & 0.58 \\
${ }^{25} \mathrm{Al}(p, \gamma){ }^{26} \mathrm{Si}$ & 0.2 & 5.514 & 2.33 & 4 & 11.7 & 0.41 & 2.48 & 0.15 \\
& 3.0 & 5.514 & 0.90 & 11 & 4.5 & 0.58 & 13.5 & 0.58 \\
${ }^{30} \mathrm{P}(p, \gamma){ }^{31} \mathrm{~S}$ & 0.2 & 6.131 & 2.16 & 4 & 10.8 & 0.49 & 3.97 & 0.15 \\
& 3.0 & 6.131 & 0.80 & 12 & 4.0 & 0.63 & 14.8 & 0.58 \\
${ }^{33} \mathrm{Cl}(p, \gamma)^{34} \mathrm{Ar}$ & 0.2 & 4.664 & 1.51 & 5 & 7.6 & 0.43 & 2.6 & 0.31 \\
& 3.0 & 4.664 & 0.61 & 14 & 3.1 & 0.59 & 14.0 & 1.19 \\
${ }^{34} \mathrm{Cl}(p, \gamma)^{35} \mathrm{Ar}$ & 0.2 & 5.896 & 1.84 & 5 & 9.2 & 0.44 & 2.7 & 0.32 \\
${ }^{37} \mathrm{~K}(p, \gamma)^{38} \mathrm{Ca}$ & 3.0 & 5.896 & 0.69 & 14 & 3.5 & 0.61 & 14.4 & 1.22 \\
${ }^{38} \mathrm{~K}(p, \gamma){ }^{39} \mathrm{Ca}(p, \gamma){ }^{66} \mathrm{Se}$ & 0.2 & 4.547 & 1.32 & 5 & 6.6 & 0.48 & 2.9 & 0.27 \\
& 3.0 & 4.547 & 0.54 & 15 & 2.7 & 0.62 & 14.6 & 1.04 \\
& 0.2 & 5.771 & 1.61 & 5 & 8.1 & 0.49 & 3.0 & 0.27 \\
& 3.0 & 5.771 & 0.61 & 15 & 3.1 & 0.64 & 15.0 & 1.06 \\
& 0.2 & 1.719 & 0.30 & 6 & 1.5 & 0.70 & 4.3 & 0.18 \\
& 3.0 & 1.719 & 0.19 & 21 & 1.0 & 0.77 & 18.4 & 0.71 \\
\hline
\end{tabular}


Table 2

Design parameters of the SECAR magnetic recoil separator.

\begin{tabular}{|l|c|}
\hline Maximum magn. rigidity $\mathrm{B} \rho$ & $0.80 \mathrm{Tm}$ \\
Minimum magn. rigidity $\mathrm{B} \rho$ & $0.14 \mathrm{Tm}$ \\
Maximum electric rigidity $\mathrm{E} \rho$ & $19.0 \mathrm{MV}$ \\
Angle acceptance, vert., horiz. & $\pm 25 \mathrm{mrad}$ \\
Energy acceptance & $\pm 3.1 \%$ \\
Mass resolution $\mathrm{R}_{H O}$ & $\approx 750$ \\
Bending radius $\rho$ & $125 \mathrm{~cm}$ \\
Beam rejection by separator & $10^{-13}-10^{-14}$ \\
Beam rejection by detector & $10^{-4}$ \\
\hline
\end{tabular}

TGT location and is being used as stand-alone system using the existing ReA3 beam from the NSCL facility awaiting the construction of SECAR and the FRIB facility. JENSA is a recirculating gas jet target system providing areal densities up to $1 \times 10^{19}$ atoms $/ \mathrm{cm}^{2}$ of hydrogen or helium isotopes in a 4-5 mm diameter cylindrical jet. In order to achieve a beam line vacuum outside of the jet chamber, differential pumping is utilized, with the size of the gas-flow-restricting apertures matched to the planned acceptance of SECAR. Only minor modifications are necessary to adapt the JENSA jet target to SECAR. In addition, an extended gas-target chamber for JENSA is planned for certain SECAR measurements where it is necessary to measure the location of a resonance inside of the target volume.

The first Section $S 1$ from the target, TGT, to the focal plane, FP1, consists of two dipole magnets, B1 - B2, and five quadrupole magnets, Q1 - Q5. Higherorder (HO) aberrations are primarily corrected by $\mathrm{HO}$ components built into the entrance and exit pole faces of the two dipoles but one hexapole magnet Hex1 is available for flexible ion-optical settings and the correction of the second-order terms to account for uncertainties in the manufacturing process. The latter can avoid costly and time-consuming retrofitting. For the same reason the quadrupole, Q1, is a combined function multipole including a variable hexapole component. Section $S 1$ accomplishes the selection of a single charge state, typically the most abundant and prepares the beam for optimum use of the Wien filter, WF1. The charge selection is accomplished in the two dipoles, B1 and B2, by magnetic analysis. Both dipoles have ports on both sides at the entrances and exits. This provides flexibility for inserting slits and other devices to stop and diagnose the beam components with different charge states and hence different magnetic rigidities. The use of two dipoles and ports allows for stopping the beam components at the best location for a wide variety of rigidities.

The second Section S2 between FP1 and FP2 consists of two dipole magnets, 


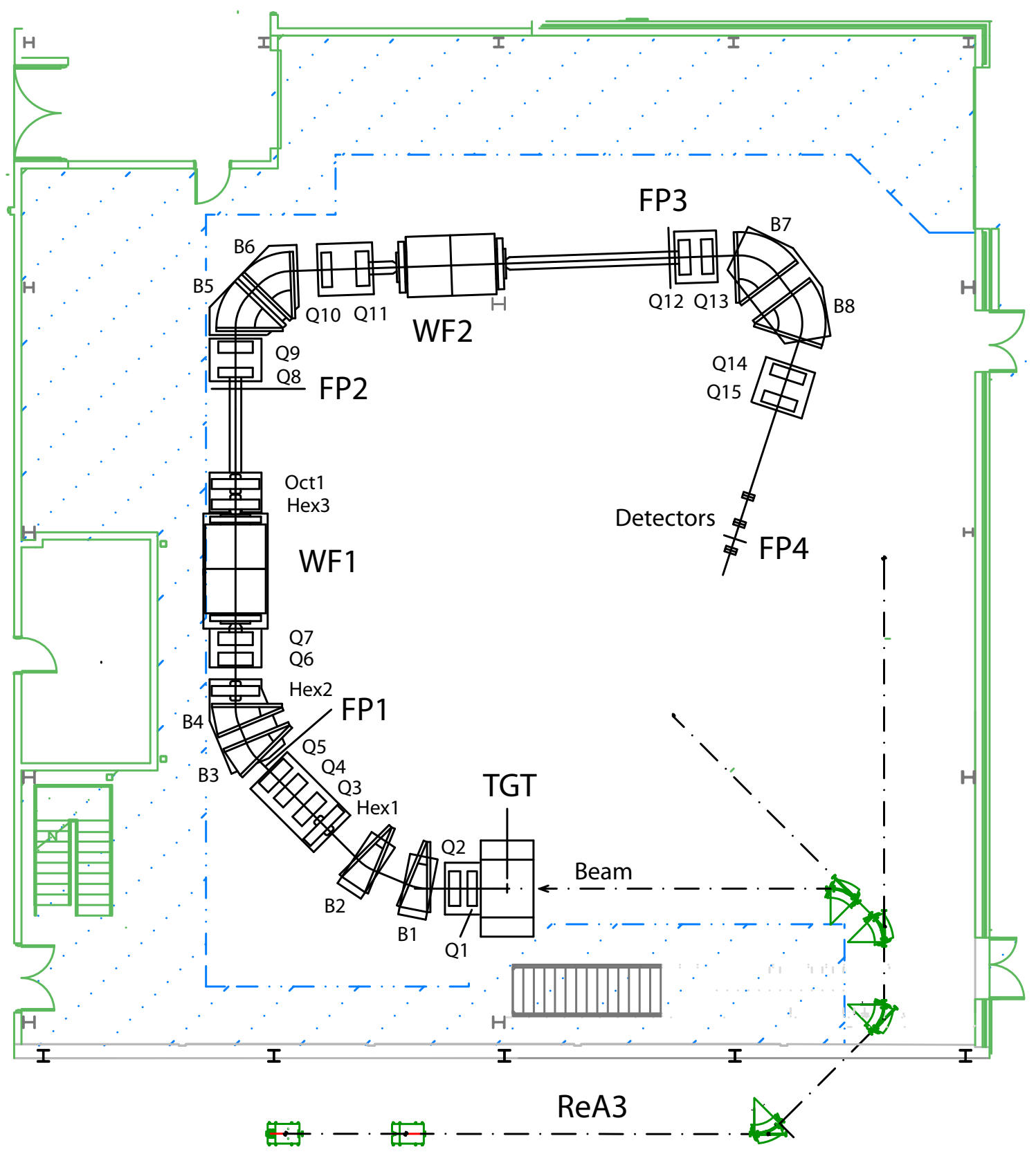

Fig. 1. SECAR floor plan with two Wien filter Sections suitable to study $(\mathrm{p}, \gamma)$ and $(\alpha, \gamma)$ reactions in inverse kinematics. The annotations are defined in the text.

B3 and B4, two quadrupole magnets, Q6 ande Q7, one Wien filter, WF1, two hexapole magnets, Hex2 and Hex3, and one octupole magnet, Oct1. After the charge state selection the beam and reaction products have essentially the same magnetic rigidity and cannot be separated by magnetic analysis. A combination of electric and magnetic dipoles is employed to separate the beam from the reaction products on the basis of their mass differences. In DRAGON, for example, separate electric and magnetic dipoles are used for this purpose. In both St. GEORGE and the Daresbury Recoil Separator Wien filters are used. A Wien filter has several advantages compared to a design 
with separate electric and magnetic bending dipole magnets. Wien filters are more compact and the magnetic and electric field ratio is chosen such that the particles with a velocity corresponding to the desired mass are not deflected and follow a straight line. This makes the ion-optics more flexible and opens up additional possibilities to optimize the recoil-separator performance.

After mass separation at FP2 a third Section S3 consisting of four quadrupoles, Q8 - Q11, two dipole magnets, B5 and B6, and a second Wien filter, WF2, identical to WF1, provides the required mass resolution $\mathrm{R}_{H O} \approx 750$ at FP3.

After the mass separation at FP3, a final momentum analysis is performed in Section 54 using magnetic dipole magnets, B6 and B7, and four quadrupoles, Q12 - Q15, to further reject background particles and to provide a clean and flexible environment for the detectors. A suitable detector system is presently under design [23] and will be constructed within the SECAR project. The mass resolution in the final detector plane is also $>750$ as in the focal plane, FP3, between the detector planes. This allows the software correction of higher order aberrations using appropriate detectors and opens the possibility of achieving a resolution as high as the resolving power of about 1300 in a similar fashion as routinely performed at high resolution spectrometers like Grand Raiden at RCNP [24] or the K600 at iThemba LABS [25].

Section $S_{4}$ also prepares the phase space of the recoil particles for optimal use of and $100 \%$ transmission through the detector system.

As will be discussed in more detail below, Section $S 3$ containing WF2 can be omitted. In this case the mass resolution is about $\mathrm{m} / \Delta \mathrm{m}=500$. While this is a cost saving measure, the major disadvantage is that the science program will be limited to masses of about $\mathrm{A}=30$ - 35 short of the $\mathrm{A}=65$ for the research program for the desired rp-process that is well matched to the RI beam available from FRIB. In this case the hardware of Section $S_{4}$ will be the same only the magnet settings will be different, so that no new hardware is required if the system is initially built without Section $S 3$ and later upgraded by adding this Section.

\section{Ion optics}

Extensive ion-optical calculations and optimizations where performed for SECAR using the computer code COSY Infinity [26] to achieve a system that meets all design requirements. A first-order design with a high mass resolving power was developed that also met all other design requirements like realistic particle ray envelopes and charge state separation. 
After establishing an optimum first order design, an extensive minimization of the $\mathrm{HO}$ aberrations was conducted. $\mathrm{HO}$ aberrations were found to be significant in the SECAR design mainly owing to the large beam envelope and the lack of ion-optical symmetry not possible because of the design functions of the Sections. For this purpose we defined a procedure to minimize the HO aberrations using the fitting capability of COSY Infinity [26]. The best would have been to perform a fit of appropriate HO magnet parameters on the basis of Monte Carlo calculations with many rays to determine and minimize the image size in the focal planes where the mass separation takes place. Since Monte Carlo calculations for many rays are too time consuming they were not practical in such fitting procedures. Therefore, we defined 189 "characteristic rays" within an "ellipsoid" given by

$$
\frac{a^{2}}{A^{2}}+\frac{b^{2}}{B^{2}}+\frac{\delta E^{2}}{E^{2}} \leq 1
$$

with the axes $\mathrm{A}=\mathrm{B}=25 \mathrm{mrad}$ and $\mathrm{E}=3.1 \%$. The following values were allowed, for $\mathrm{A}: 0, \pm 8.333, \pm 16.666$, and $\pm 25 \mathrm{mrad}$, for $\mathrm{B}: 0, \pm 25 \mathrm{mrad}$, for $\mathrm{E}$ : $0, \pm 1.55, \pm 3.1 \%$. In addition the object sizes of the beam at the target where allowed to assume the values $\mathrm{X}=\mathrm{Y}=0, \pm 0.0075 \mathrm{~m}$. This is larger than the predicted beam size of $0.0050 \mathrm{~m}$ of the re-accelerated ReA3 beam to allow for a somewhat larger than predicted beam size.

For design purposes, the assumption was made that the 189 characteristic rays realistically represent the envelopes and the image sizes of the beam. The validity of this procedure was then tested by performing a many-ray Monte Carlo calculation with the final HO magnet components and settings and by comparing the image size with those obtained using the 189 characteristic rays.

Fig. 2 show the ion optics of the SECAR layout presented in Fig. 1, optimized and corrected up to order 4 . The upper and lower panel show the horizontal and the vertical plane, respectively. All 189 characteristic rays are shown. By design, the horizontal envelope is relatively large in the first and second sections Section $S 1$ and Section S2, while the vertical envelope never exceeds $\pm 6 \mathrm{~cm}$ to keep the costly dipole gap size small. Also a large envelope in the Wien filters is required for a large mass separation. The system has an achromatic $\left(\mathrm{x} \mid \delta_{E}\right)=0,\left(\mathrm{a} \mid \delta_{E}\right)=0$, focus $\left(\mathrm{x} \mid x^{\prime}\right)=0$ at $\mathrm{F} 2$ and a zero dispersion $\left(\mathrm{x} \mid \delta_{E}\right)=0$ focus at $\mathrm{F} 3$. The HO aberrations are carefully corrected up to order 4 to achieve the best possible mass resolution.

Fig. 2 shows a left-right asymmetry of the envelope about the central ray. This is mainly an effect of the second and third order aberrations. More symmetric envelopes were found allowing a reduction of the horizontal good-field region, but this resulted invariably in a reduced mass resolution. Since the highest possible mass resolution is paramount, we decided to accept the asymmetric 
envelope.

The mass resolution of SECAR is $\approx \mathrm{R}_{H O}=510$ and 780 at the focal planes FP2 and FP3 after the first and second Wien filter, respectively. For a mass of $\mathrm{A}=65$ this translates in a distance $\mathrm{D}$ between beam and reaction products of $\mathrm{D}=8.0 \sigma$ and $12.1 \sigma$, assuming $\sigma$ is the maximum of the calculated deviations in horizontal direction of the 189 characteristic rays and a $\operatorname{NBR}(8.0 \sigma)=1.3$ $10^{-9}$ and $\operatorname{NBR}(12.1 \sigma)=4.416^{-24}$, respectively. It should be noted that the NBR depends strongly on the integration interval.

The first section Section $S 1$ allows the selection of the desired charge state. Two examples of charge states different by $\Delta q / q=1 / 15$ and $1 / 33$ from the recoils are shown. The most demanding, calculated case of adjacent charge states of fully stripped ${ }^{65}$ As ions with charge differences $\Delta q / q=1 / 33$ are well separated in the focal plane, FP1, in front of dipole, B3. In reality at the low beam energies where SECAR will operate, the ions are never fully stripped, so that charge state separation is always better than this calculated case. Beam components with charge states different by $\Delta q / q>1 / 15$ are already separated in front of hexapole Hex1, where e.g. slits installed in the provided ports at the entrance and exit of dipole B2 vacuum chambers may be used to stop all but the selected charge states.

In the final Section $S_{4}$, from focal plane, FP3, to the detector plane, FP4, the optics is adjusted to provide a $150 \mathrm{~cm}$ long TOF path length with two 30 $\mathrm{mm}$ diameter detectors at the start and end of the flight path. There is also a tight waist in horizontal and vertical direction at the exit of dipole, B7, where a cleanup slit can be inserted to reduce the background.

\section{Transmission and background estimation simulations}

In order to determine if the proposed ion-optical solution meets the transmission and good rejection requirements, we performed Monte Carlo simulations. While the transmission simulation are relatively straighforward, the estimation of possbile background sources is not. We used a method inspired by the calculation performed for the design of the DRAGON recoil separator [?]. In the following we describe the simulation of the transmission efficiency in section 5.1 and we use the simulated particle trajectories as sources of possible background in section 5.2 . 


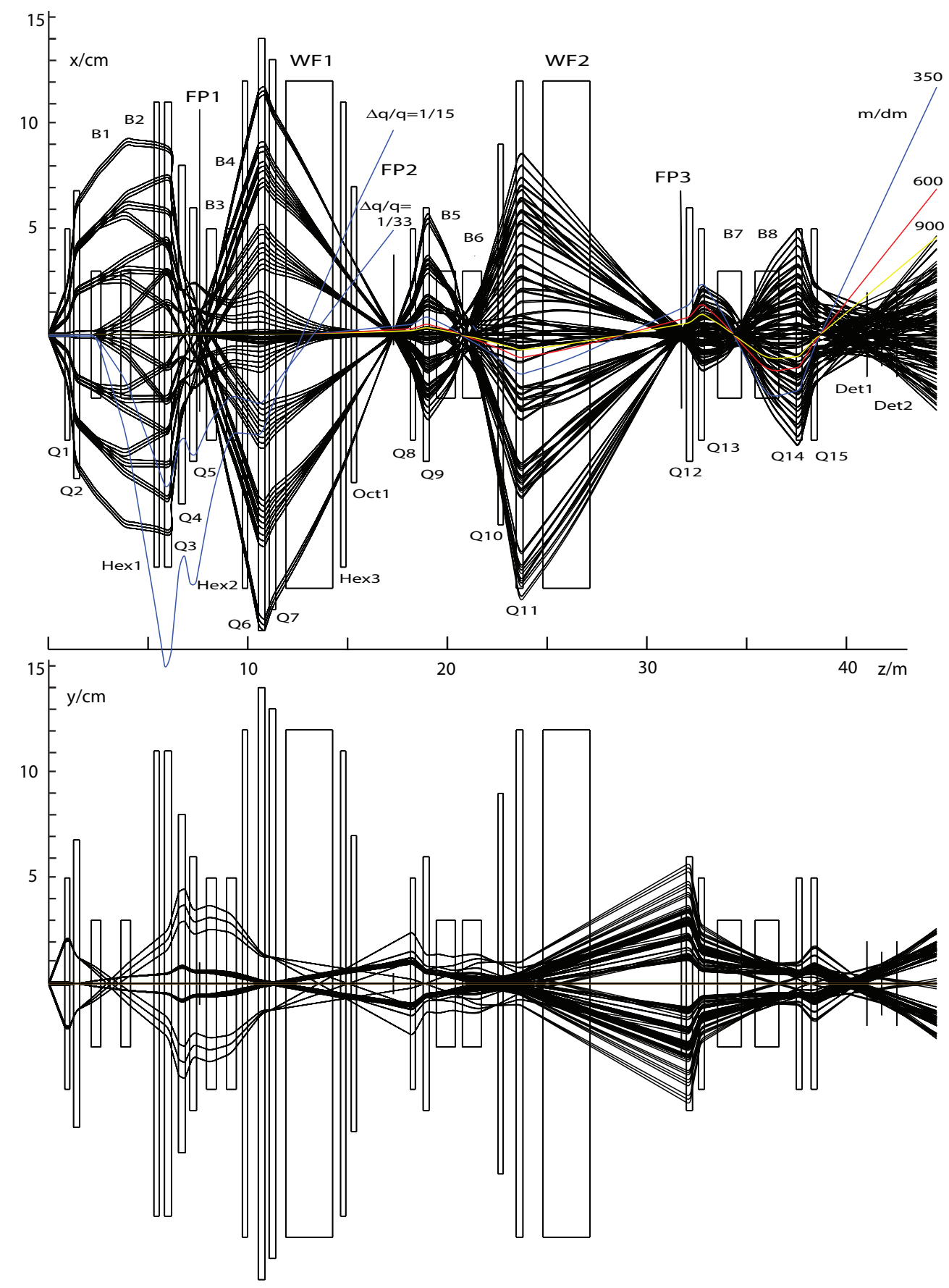

Fig. 2. The ion optics of SECAR for the layout shown in Fig. 1 corrected up to order 4. Shown are 189 characteristic rays in the horizontal and vertical planes in the upper and lower panel, respectively. The mass resolutions in the horizontal plane at $\mathrm{FP} 2$ is $\mathrm{m} / \Delta \mathrm{m}=510$ and at FP3 $\mathrm{m} / \Delta \mathrm{m}=780$.

\subsection{Transmission efficiency simulations}

In order to simulate the transmission efficiency, we choose three reactions characteristic for the SECAR program at various energies: ${ }^{15} \mathrm{O}(\alpha, \gamma){ }^{19} \mathrm{Ne}$ at 
$E_{C M}=0.3 \mathrm{MeV} / u,{ }^{21} \mathrm{Na}(p, \gamma)^{22} \mathrm{Mg}$ at $E_{C M}=0.3 \mathrm{MeV} / u$ and $1 \mathrm{MeV} / u$, and ${ }^{65} \mathrm{As}(p, \gamma){ }^{66} \mathrm{Se}$ at $E_{C M}=1 \mathrm{MeV} / u$ and $3 \mathrm{MeV} / u$. A realistic beam from the ReA3 accelerator [17] was generated and a Geant4 [18] simulation of its interaction in the JENSA gas target, with a thickness of $10^{18}$ atoms $/ \mathrm{cm}^{2}$, was used to obtain the needed ion optical properties of the beam and recoil particles as they enter SECAR. The recoil generation assumed a uniform cross section along the longitudinal direction of the gas target. About $510^{7}$ beam and 5 $10^{6}$ recoil particles were generated for each of the selected reactions.

The results of the Geant4 simulation was then used as an input for a COSY INFINITY calculations. All particles with all possible charge states were transported through SECAR and their properties recorded at the exit of every optical element (drifts, quadrupoles, dipoles, Wien filters, etc). The transmissions of the recoils of the selected charge states all the way to the focal plane detector are presented in Table 3. For the same charge state, the beam particle simulated are stopped at the mass slits at FP2 in Fig. 1) downstream of the first Wien filter (WF1 in Fig. 1). Beam particles with one charge state difference are properly stopped at the charge selection slits (FP1 Fig. 1) or even further upstream for larger $\Delta q / q$. For example rays with extreme $\Delta q / q=1 / 15$ and $1 / 33$ see Fig. 2. The later will be caught in the slits installed in the dipole magnets chambers (see Section 7.1).

Table 3

Transmission efficiency of the recoils for the 5 characteristics reactions

\begin{tabular}{|l|c|}
\hline Reaction & Transmission efficiency \\
\hline${ }^{15} \mathrm{O}(\alpha, \gamma)^{19} \mathrm{Ne}$ at $E_{C M}=0.3 \mathrm{MeV}$ & $95 \%$ \\
${ }^{21} \mathrm{Na}(p, \gamma)^{22} \mathrm{Mg}$ at $E_{C M}=0.3 \mathrm{MeV}$ & $96 \%$ \\
${ }^{21} \mathrm{Na}(p, \gamma)^{22} \mathrm{Mg}$ at $E_{C M}=1 \mathrm{MeV}$ & $97 \%$ \\
${ }^{65} \mathrm{As}(p, \gamma){ }^{66} \mathrm{Se}$ at $E_{C M}=1 \mathrm{MeV}$ & $97 \%$ \\
${ }^{65} \mathrm{As}(p, \gamma){ }^{66} \mathrm{Se}$ at $E_{C M}=3 \mathrm{MeV}$ & $98 \%$ \\
\hline
\end{tabular}

\subsection{Background simulations}

Despite the high transmission efficiency of the simulations, it is clear that some unreacted beam particles will pass beyond the mass slit at FP2. The number of simulated beam particles is not sufficient to reach sufficient tail statistics to determine the beam rejection. However, using the estimate presented in section 5.1 and equation (1) the rejection is better that $10^{10}$. In addition to the tails, we must consider particles scattered off residual gas molecules and the separator component surfaces, and the projectiles that undergo charge changes from the originally selected charge states.

To address these issues we performed Monte Carlo transport simulations of 
charge-changing and scattering events to identify and as much as possible eliminate weaknesses of our conceptual design. Based on experience with recoil separators, these effects are especially important sources of background. According to the experience with the DRAGON recoil separator [5][24], the rejection of projectiles becomes worse at higher target gas pressures, at higher pressures in their first stage ED/MD system, and at lower bombarding beam energies. For the ERNA recoil separator used to measure alpha-particle capture using stable beams [9][25], a major background source was projectile scattering off the component surfaces, especially projectiles with charge states differing from the selected recoil charge state. ERNA was recently moved from an accelerator laboratory in Bochum, Germany to the laboratory in Caserta, Italy. An additional dipole magnet was added immediately after the gas target in order to attempt to reduce this background [9][25].

To reduce computational requirements and allow for the simulation of an as large as possible number of particles, we performed our projectile rejection simulations initially for a system with just one Wien filter (see Section 9). This system includes all types of separation elements used in the full system (charge state selection, Wien filter based mass separation, and a dipole-based cleanup Section $S_{4}$ ) and, therefore, allows the testing of the performance of each element. This setup is particularly useful to test the performance of the cleanup Section $S_{4}$ as more simulated particles will reach that magnet section in the 1-Wien filter system. The subset of particles passing through the Wien filter stage in this simplified model can then be investigated in terms of their fate in the more complicated 2-Wien filter system in a much more efficient way.

At first we identified critical regions of phase space after each separator component that contains trajectories that can reach the final focal plane. This information was obtained by tracing ion trajectories backwards through the system starting at the focal plane. Once these phase space windows were identified, we used forward Monte Carlo simulations to generate scattering events or charge-changing events at multiple locations along each trajectory. At each event a large number of charge state changes, angle changes, and energy changes was generated, and each of these new trajectories was then compared to the phase space window that would lead to transmission to the focal plane at the respective location where the trajectory is generated. We used a conservative approach in our phase-space calculations leading to the selection of particle trajectories that may not reach the final focal plane. Therefore, we simulated the transport of the particles that fall into the critical phase space regions with a full COSY Infinity 4th order model. This approach reduces the unrealistically large number of particles that would need to be transported in a traditional simulation.

Different methods were used to alter the particle properties for simulating 
charge-changing and scattering interactions. For charge changing events, we used the calculated transmissions and fixed all properties except for the charge state that was changed by 1 and 2 units from its initial value assuming cross sections for losing or gaining more than two electrons are negligible. For scattering interactions, we used a more complex methode that changes the energy and angle of the tranmitted particle but not the position or charge. We considered elastic scattering off five target nuclei: ${ }^{1} \mathrm{H}$ and ${ }^{4} \mathrm{He}$ (gas target), ${ }^{16} \mathrm{O}$ (water), ${ }^{27} \mathrm{Al}$ (beam pipe), and ${ }^{48} \mathrm{Ti}$ (Wien filter electrodes). The calculations were performed for 5000 different combinations of energies and angles for each particle, using 40 bins within the polar angle range of $0 \mathrm{deg}<\Theta<30 \mathrm{deg}$ and 125 bins within the azimuthal angle range of $0 \leq \phi \leq 2 \pi$. The energy of a particle after scattering at a given angle is calculated assuming elastic scattering. Additionally, for beam particles lighter than the scattering targets, we considered both possible kinematic solutions corresponding to the forward and backward direction in the center of mass system. Each of those scattering combinations (angle, energy) was combined with the particle's position, velocity, and charge values to generate a set of altered particle properties that were recorded if they fell within a critical phase space region or ignored if they did not.

A plot of the number of particles that can possibly reach the focal plane detector after a charge-changing interaction as a function of the position along the ion-optical axis of the separator is presented in Fig. 3 for ${ }^{21} \mathrm{Na}$ ions from the ${ }^{21} \mathrm{Na}(p, \gamma){ }^{22} \mathrm{Mg}$ reaction. This figure also gives a similar plot for scattered ${ }^{65} \mathrm{As}$ ions from the ${ }^{65} \mathrm{As}(p, \gamma){ }^{66} \mathrm{Se}$ reaction. We find that the Wien filter volume is the source of particles that, if they change charge state, potentially, can reach the detector. The first $10 \mathrm{~m}$ of the separator is the source of possible scattering events reaching the final focal plane. We denote these as possibly reaching the focal plane because of the conservative approach we took in generating the critical phase space regions.

We, therefore, employed $4^{\text {th }}$ order SECAR transfer matrixes to determine if the particles identified indeed reach the focal plane. In Fig. 4 we present particle trajectories in the horizontal plane of ${ }^{21} \mathrm{Na}^{8+}$ ions from the ${ }^{21} \mathrm{Na}(\mathrm{p}, \gamma)^{22} \mathrm{Mg}$ reaction that undergo a charge-changing interaction in the Wien filter and are scattered into the critical phase space. When the particle trajectories are traced, however, it becomes clear that these ions do not proceed beyond the focal plane FP2. The figure also shows ${ }^{65} A s^{25+}$ ions from the ${ }^{65} A s(p, \gamma){ }^{66} S e$ reaction that undergo scattering inside quadrupole magnet Q3. These particles also clearly do not reach the detector plane of the single Wien filter system.

The yield of charge-changing events on residual gas molecules along SECAR can be calculated using a phenomenological estimate of the cross section [26] and the calculated vacuum pressure distribution in the separator. The yield is smaller than $10^{-6}$. It is the highest for ${ }^{15} \mathrm{O}+{ }^{16} \mathrm{O}\left(<10^{-5}\right)$, while it is the lowest 
for ${ }^{65} \mathrm{As}+{ }^{4} \mathrm{He}\left(<10^{-9}\right)$. An estimate of the scattering yield on solid surfaces (vacuum chamber walls, slits, and Wien filter electrodes) along the separator gives a yield in the range of $10^{-6}$ to $10^{-3}$. Based on these yields, we conclude that the absence of any events reaching the focal plane for capture reactions of lighter $\mathrm{A}=21$ beams from simulations with $4.5 \times 10^{7}$ scattered or chargechanged particles at each scattering or charge-changing location demonstrates a rejection of the order of $10^{13}$ for single charge changing events, $10^{12}$ for single scattering on residual gas molecules, and $10^{10}$ for single scattering off the chamber walls.

However, the situation is different for reactions with $\mathrm{A}=65$ beams. While Fig. 4 shows that ${ }^{65} A s^{25+}$ ions from the ${ }^{65} A s(p, \gamma){ }^{66}$ Se reaction undergoing scattering in quadrupole magnet Q3 do not reach the focal plane of the single Wien filter system, the situation is different for ${ }^{65} A s^{25+}$ ions from the same reaction undergoing a charge-changing interaction in the Wien filter (see Fig. 5). These beam particles clearly do reach the focal plane. While most of them miss the detector location in the limited sample size of our simulations $\left(4.5 \times 10^{7}\right.$ ions) because the cleanup Section $S_{4}$ deflects them, we cannot be certain that this holds true for a more realistic case of only one recoil for every $10^{13}$ projectiles. While this figure shows the effectiveness of the cleanup Section $S_{4}$ in the single WF design to reject some of the charge-changed particles, it also demonstrates that the second Wien filter is needed to reject projectiles even at the $10^{7}$ level for measurements of reaction rates with heavier beams.

Our investigation of particles created by charge change or scattering demonstrates that the system deals effectively with these major sources of leaky beam. A single Wien filter system with a cleanup Section can deal with single charge changing or scattering effects efficiently, except for reaction measurements with heavier beams. However, multiple scattering effects that are beyond current simulation capabilities, but are likely a significant source of leaky beam, may still be a problem. The second Wien filter stage is clearly needed to reject higher-mass projectiles and to dramatically reduce multiple scattering or charge-changing events. Our simulations also demonstrated the efficacy of our cleanup Section.

\section{Magnet Design}

The ion-optical design of the separator determines the properties of the dipole, quadrupole and HO magnets. They have to be designed and built so that the good-field regions in both transverse directions and the necessary field strengths meet the ion-optical requirements.

All magnets are excited by highly stabilized power supplies and are designed 


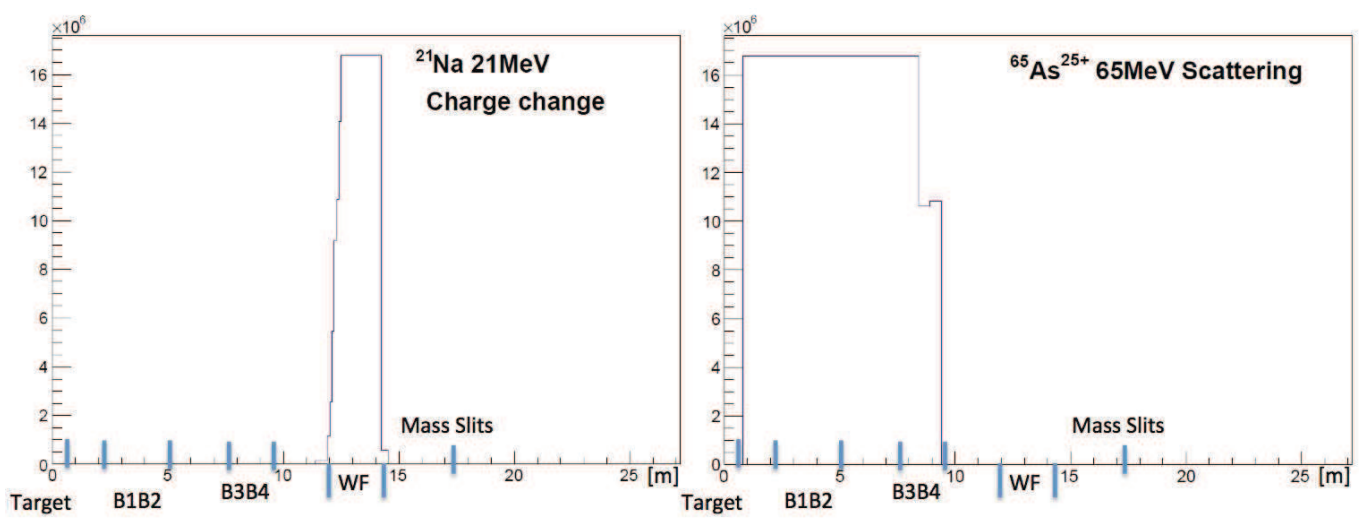

Fig. 3. Number of particles that can possibly reach the SECAR focal plane as a function of position along the ion-optical axis. Left side panel: ${ }^{21} \mathrm{Na}$ ions from the ${ }^{21} \mathrm{Na}(\mathrm{p}, \gamma)^{22} \mathrm{Mg}$ reaction undergoing a charge-changing interaction are primarily generated in the Wien filter (labeled WF). Right side panel: ${ }^{65} \mathrm{As}$ ions from the ${ }^{65} \mathrm{As}(\mathrm{p}, \gamma){ }^{66} \mathrm{Se}$ reaction undergoing a scattering interaction are primarily generated upstream of the first Wien filter. Locations of the target, dipole magnets, and first Wien filter are indicated along the ion-optical axis.
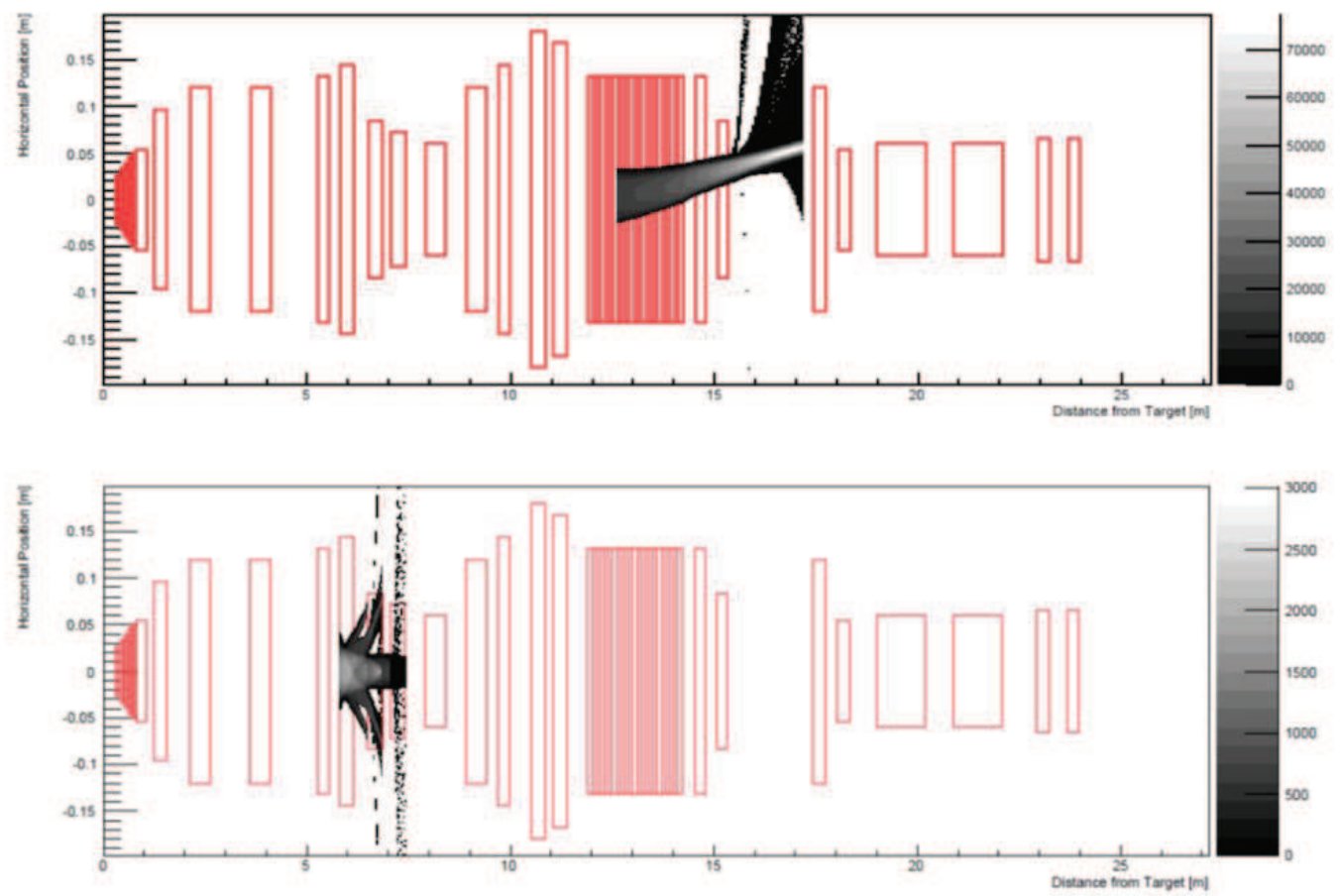

Fig. 4. Particle trajectories in the horizontal plane. Top: ${ }^{21} \mathrm{Na}^{8+}$ ions from the ${ }^{21} \mathrm{Na}(\mathrm{p}, \gamma){ }^{22} \mathrm{Mg}$ reaction undergoing a charge-changing interaction in the Wien filter do not proceed beyond focal plane FP2. Bottom: ${ }^{65} \mathrm{As}^{25+}$ ions from the ${ }^{65} \mathrm{As}(\mathrm{p}, \gamma){ }^{66}$ Se reaction undergoing scattering in quadrupole Q3. These particles also do not reach the detectors in the final focus of the single Wien filter system. 


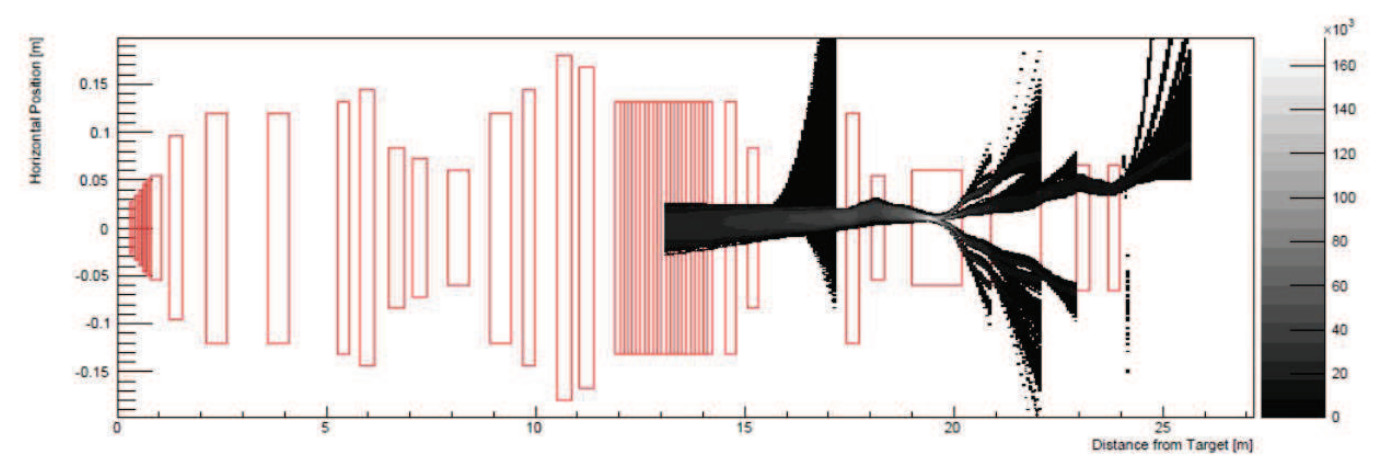

Fig. 5. Trajectories in the horizontal plane of ${ }^{65} \mathrm{As}^{25+}$ ions from the ${ }^{65} \mathrm{As}(\mathrm{p}, \gamma){ }^{66} \mathrm{Se}$ reaction undergoing a charge-changing interaction in the Wien filter. These particles do reach the final focus of the single Wien filter system.

to operate at fields up to a maximum of $0.64 \mathrm{~T}$ in the gap of the dipoles and $0.45 \mathrm{~T}$ at the pole tips of the quadrupoles to avoid saturation of the magnet iron. All iron pole pieces and return yokes are machined of solid, soft magnet iron. The coils are normal conducting with hollow-copper conductors to allow water cooling. The coil temperatures are kept below $55^{\circ} \mathrm{C}$. All magnets include vacuum chambers with ports to provide the required high vacuum and allow access for pumping, slits and other diagnostics elements.

\subsection{Dipole Magnets}

The eight dipoles are H-type magnets. They will be manufactured according to the specifications given in Table 4 . All dipole magnets have bending radii of $1250 \mathrm{~mm}$. The Good-Field-Region (GFR) is defined by the region of field deviations $\mathrm{dB} / \mathrm{B}< \pm 0.02 \%$. As an example the midplane view of dipole, $\mathrm{B} 1$, is shown in Fig. 6. The figure also shows the vacuum chamber with access ports at the entrance and exit of the magnet allowing the installation of a variety of slits and diagnostic elements to stop and measure the beam or background, as may be required for a particular experiment. An additional port extended through the middle of the inner yoke allows the insertion of a NMR or Hall probe for precise field setting and monitoring. For cost reasons, easy access to the inside of the vacuum chambers, and to avoid magnetization during welding, seals are provided by Viton O-rings. All magnets have $0^{\circ}$ ports in both directions for alignment and other access needs that may arise.

In order to meet the specifications, in particular the large design acceptances, HO aberrations have been corrected up to order 4. The HO order corrections were mainly provided by shaping the field boundaries at the entrances and exits of the dipole magnets. In accordance with the COSY notation [26] the 


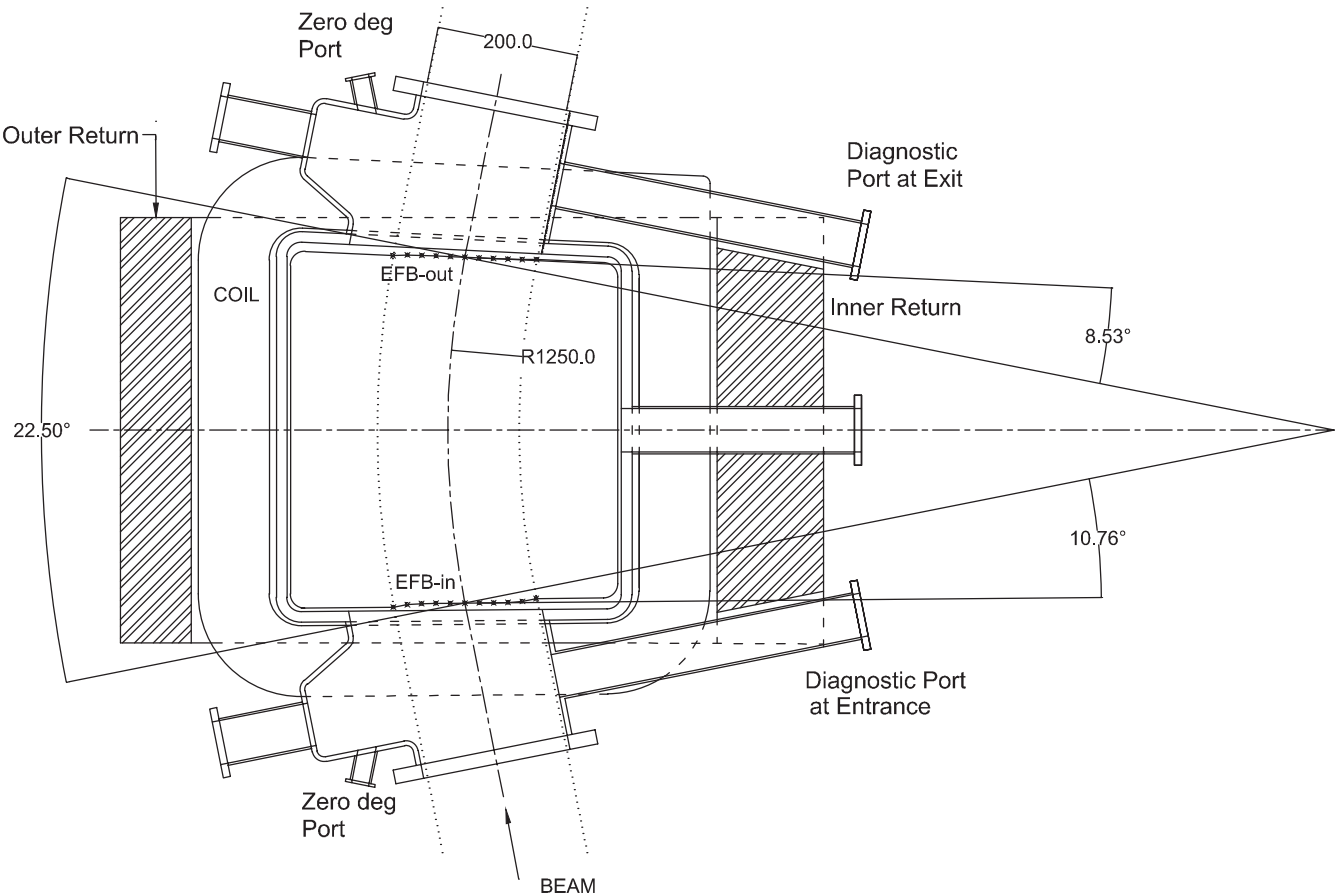

Fig. 6. View from above the midplane of the conceptual design of dipole magnet, $\mathrm{B} 1$, and the vacuum chamber. The physical shape of the pole piece at the entrance (EFB-in) and the exit (EFB-out) are designed to create the higher order corrections. All dimensions are in millimeter and degree.

boundaries are parameterized by polynomials for the

$$
\text { entrances } \quad z(x)=\sum_{i=1}^{5} s_{1 i} \cdot x^{i} \quad \text { and the exits } \quad z(x)=\sum_{i=1}^{5} s_{2 i} \cdot x^{i} \text {. }
$$

Here $\mathrm{z}(\mathrm{x})$ is the displacement in meter (positive is inwards into the magnet) at the position $\mathrm{x}$ in meter, where $\mathrm{x}$ is the transverse coordinate with respect to the optical axis, with the origin where it crosses the field boundary. The coefficients of the optimized field boundaries are listed in Table 4. The first order coefficients $s_{11}$ at the entrances and $s_{21}$ at the exits are the tangents of the edge rotation and provide vertical focusing for positive coefficients. This focusing helps to minimize the vertical particle envelopes and, therefore, the gaps of the dipole magnets. It also reduces the maximum required focusing strengths of the quadrupole magnets. As an example of the shape of the edge shape at the exit and entrance a drawing of dipole magnet, B1, is shown in Fig. 6. In order to be able to set the magnetic fields reproducibly, all dipoles have ports where NMR or precision Hall probes can be inserted as shown in the figure.

For the quadrupole and $\mathrm{HO}$ edge parameters without decimal points only those non-zero digits are shown that effect the mass resolution of about $\mathrm{m} / \Delta \mathrm{m}=$ 0.01 or more. For numbers with decimal points these are the rightmost digits 
Table 4

Design parameters of the dipole magnets. The following parameter are identical for all dipole magnets: bending radius $=1250 \mathrm{~mm}$, maximum rigidity $=0.80 \mathrm{Tm}$, minimum rigidity $=0.14 \mathrm{Tm}$, maximum dipole field $=0.64 \mathrm{~T}$, tolerance in GoodField Regions (GFR) dB/B $= \pm 0.02 \%$. The parameters $\mathrm{s}_{k i}$ are defined in equation (3).

\begin{tabular}{|l|c|l|l|l|l|l|l|l|l|}
\hline & & \multicolumn{9}{|c|}{ Dipole Magnet } \\
\hline & Units & B1 & B2 & B3 & B4 & B5 & B6 & B7 & B8 \\
\hline \hline Horiz.GFR & $\mathrm{mm}$ & 200 & 200 & 100 & 200 & 100 & 100 & 100 & 100 \\
\hline $\begin{array}{l}\text { Bending } \\
\text { angle }\end{array}$ & deg & 22.5 & 22.5 & 22.5 & 22.5 & 42.5 & 42.5 & 55.0 & 55.0 \\
\hline $\begin{array}{l}\text { Arc } \\
\text { length }\end{array}$ & $\mathrm{mm}$ & 490.9 & 490.9 & 490.9 & 490.9 & 927.2 & 927.2 & 1199.9 & 1199.9 \\
\hline Vert. gap & $\mathrm{mm}$ & 60 & 60 & 112 & 112 & 72 & 72 & 60 & 60 \\
\hline Pole width & $\mathrm{mm}$ & 380 & 380 & 400 & 500 & 300 & 300 & 280 & 280 \\
\hline Entr. $\mathrm{s}_{11}$ & & 0.1900 & 0.1150 & 0.1900 & 0.1900 & 0.1890 & 0.1970 & 0 & 0 \\
\hline Entr. $\mathrm{s}_{12}$ & $1 / \mathrm{m}$ & 0.0025 & 0.0125 & 1.07 & -0.339 & 0.696 & -1.66 & 0 & 0 \\
\hline Entr. $\mathrm{s}_{13}$ & $1 / \mathrm{m}^{2}$ & 0.154 & 0.198 & -9.10 & -5.51 & -0.953 & -50.0 & 0 & 0 \\
\hline Entr. $\mathrm{s}_{14}$ & $1 / \mathrm{m}^{3}$ & 0.78 & -40.77 & 0.0 & -0.84 & -53. & 0 & 0 & 0 \\
\hline Exit $\mathrm{s}_{21}$ & & 0.1500 & 0.1150 & 0.1150 & 0.1900 & -0.172 & 0.200 & 0 & 0 \\
\hline Exit $\mathrm{s}_{22}$ & $1 / \mathrm{m}$ & -0.019 & -0.2448 & 0.0410 & -0.030 & -5.928 & -4.00 & 0 & 0 \\
\hline Exit $\mathrm{s}_{23}$ & $1 / \mathrm{m}^{2}$ & 0.147 & 1.411 & 32.7 & -0.364 & -26.5 & 69. & 0 & 0 \\
\hline Exit $\mathrm{s}_{24}$ & $1 / m^{3}$ & 0.10 & 37.47 & -57. & -0.15 & 940. & 0 & 0 & 0 \\
\hline
\end{tabular}

shown. The acceptable tolerances that have negligible effect on the resolution are always larger. Typically deviations of $0.002,0.01 \mathrm{~m}^{-1}$, and $0.1 \mathrm{~m}^{-2}$ for the quadrupole, hexapole and octupole parameters, respectively, are acceptable and do not affect the performance of the system.

\subsection{Quadrupole Magnets}

The 15 quadrupole magnets of the separator system will be manufactured according to the specifications in Table 5 . Most quadrupole magnets require the dimensions of the horizontal GFR to be significantly wider than the vertical GFR. In these cases the vacuum chambers are wider in the horizontal direction as shown schematically in Fig. 7. This allows a GFR that is up to about $20 \%$ larger than the diameter of the quadrupole.

All dipoles will be equipped with precision Hall probes to accurately set the dipole field. The effects of hysteresis of the quadrupoles on the resolution are 
Table 5. Design parameters of the quadrupole magnets. The maximum inhomogeneity, defined as deviation from the design gradient, is $0.2 \%$ for all quadrupoles.

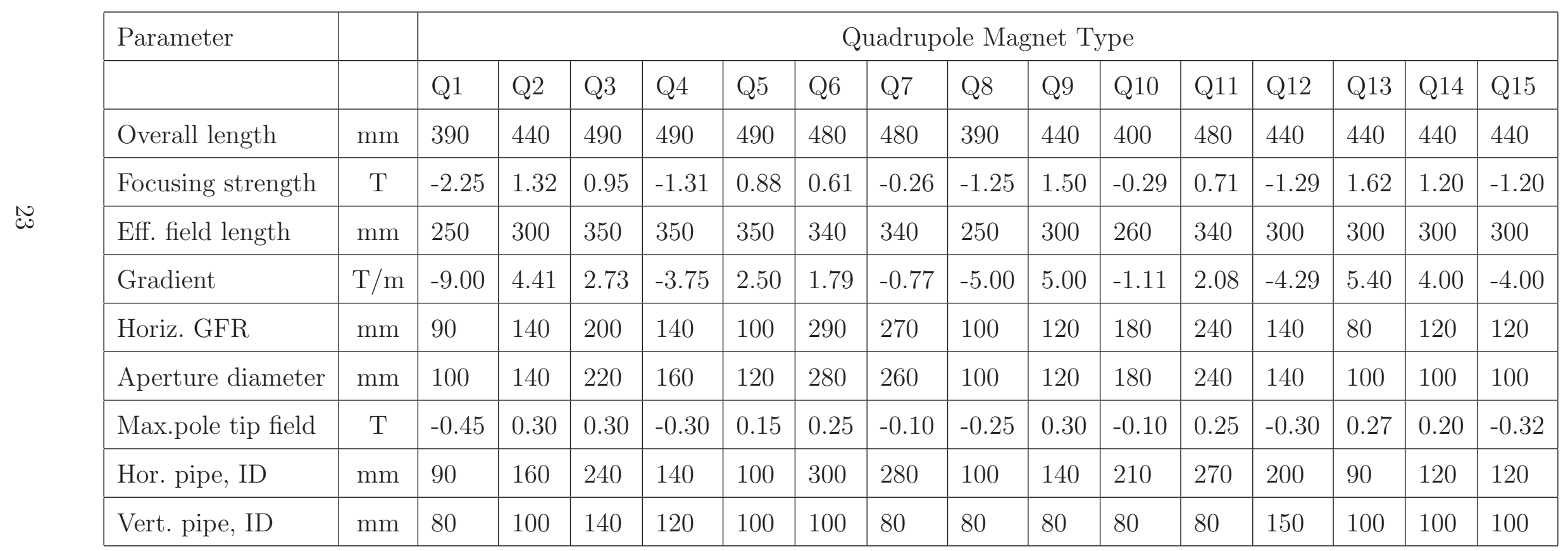




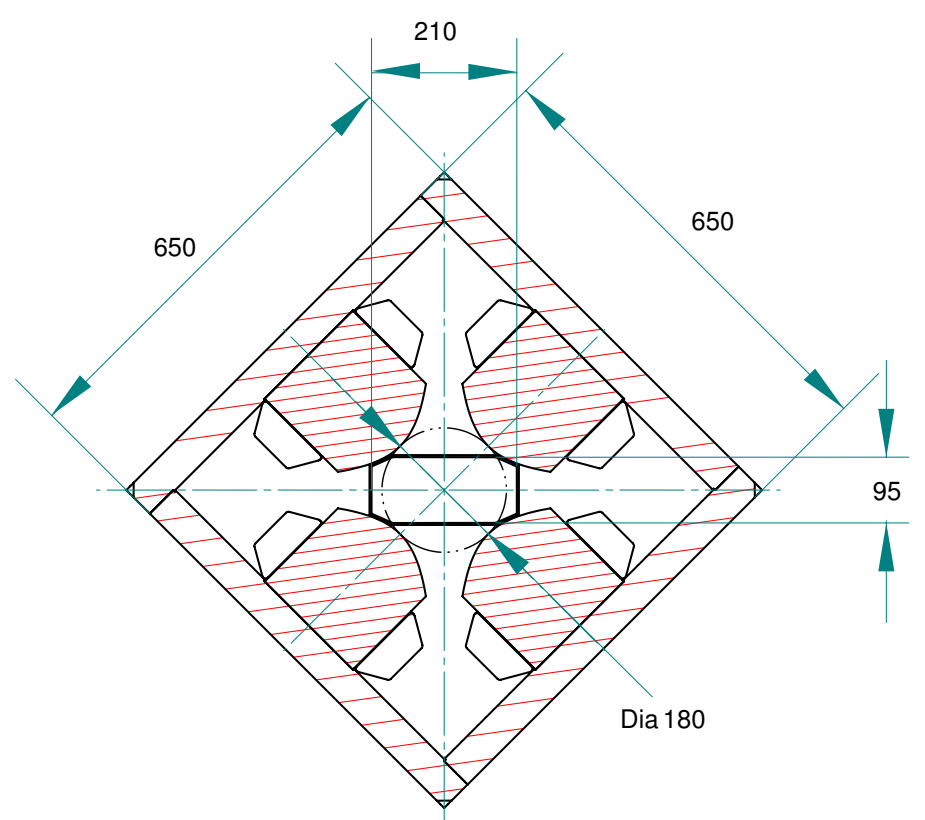

Fig. 7. Conceptual cross section drawing of the quadrupole magnet Q10 including the vacuum chamber.

small enough so that the quadrupole fields can be set by current.

\section{Design of the Wien filters}

The Wien filters (WFs) are the most sophisticated and fragile devices in the entire SECAR system. They provide the required mass separation, and their reliable operation is absolutely critical to achieving the required performance of the recoil separator. Their compact design poses several technical challenges. These result from the fact that the electric dipole is embedded in a magnet dipole.

\subsection{Design Parameters}

As discussed above, we have designed our system with a mass resolution of 510 at FP2 after the first Wien filter, and a mass resolution of 780 at FP3 after the second Wien filter to achieve a high suppression of beam projectiles. This requires high field strengths to significantly deflect the projectiles and this means high voltages on electrodes. The electrodes and their enclosing vacuum chamber need to be large to hold high voltage and to have a large electric GFR. However, this electrostatic system must be installed inside the gap of the dipole magnet. With increasing size, the electrostatic system quickly drives the size of the magnet to impractical dimensions to maintain a magnetic GFR that 
overlaps with the electric GFR. Our design maximizes the electric GFR and minimizes the surrounding magnet size while maintaining an excellent overlap of the magnetic and electric GFRs. Also, the fields have to be stable in time so that no unwanted recoil deflections occur. This places tight constraints on the means of supplying HV to the WF electrodes, and also on our approach to ensure that no sparking occurs during normal operation.

These requirements place some stringent requirements on the electrostatic properties of the velocity filters, in particular on the horizontal electrode gap, the vertical GFR, the field gradient, and the high voltage. These and other design parameters are summarized in Table 6

Table 6

Design parameters of Wien filter.

\begin{tabular}{|c|c|c|c|}
\hline \multicolumn{4}{|c|}{ SECAR Wien Filter } \\
\hline $\begin{array}{l}\text { Good-field } \\
\text { region (GFR) }\end{array}$ & $\begin{array}{l}\text { Horizontal } \\
\text { Vertical }\end{array}$ & $\begin{array}{l}\mathrm{mm} \\
\mathrm{mm}\end{array}$ & $\begin{array}{l} \pm 110 \\
\pm 35\end{array}$ \\
\hline $\begin{array}{l}\text { Dipole } \\
\text { magnet }\end{array}$ & $\begin{array}{l}\text { Max. B field } \\
\text { Effective field length } \\
\text { Pole gap, vertical } \\
\text { Pole width } \\
\text { B field, homogeneity } \\
\text { Estimated power } \\
\text { Iron weight } \\
\text { Weight of } 2 \text { coils }\end{array}$ & $\begin{array}{l}\mathrm{T} \\
\mathrm{mm} \\
\mathrm{mm} \\
\mathrm{mm}\end{array}$ & $\begin{array}{l}0.12 \\
2365 \\
900 \\
1020 \\
\pm 0.0002 \text { in GFR } \\
50 \\
12800 \\
2300\end{array}$ \\
\hline $\begin{array}{l}\text { Electrostatic } \\
\text { system }\end{array}$ & $\begin{array}{l}\text { Max. E field } \\
\text { Max. Voltage on electrodes } \\
\text { Effective field length } \\
\text { Electrode gap, horizontal } \\
\text { Electrode height, vertical } \\
\text { E field, homogeneity } \\
\text { Electrode to grounded chamber } \\
\text { Max. E field in gap to wall } \\
2 \text { Electrodes, Ti, non magn. } \\
\text { Vacuum chamber, SS, non magn. }\end{array}$ & $\begin{array}{l}\mathrm{kV} / \mathrm{mm} \\
\mathrm{kV} \\
\mathrm{mm} \\
\mathrm{mm} \\
\mathrm{mm} \\
\mathrm{mm} \\
\mathrm{kV} / \mathrm{mm} \\
\mathrm{kg} \\
\mathrm{kg}\end{array}$ & $\begin{array}{l}2.7 \\
\pm 300 \\
2365 \\
\pm 110 \\
538 \\
\pm 0.0002 \text { in GFR } \\
141 \\
3.8 \\
\text { approx. } 1200 \\
\text { approx. } 3300\end{array}$ \\
\hline
\end{tabular}




\subsection{Layout}

SECAR has two identical Wien filters. The cross section in the center of the Wien filters perpendicular to the central ray is schematically shown in Fig. 8. The recoil particles enter the system in the center of the GFR of vertically 70 $\mathrm{mm}$ and horizontally $220 \mathrm{~mm}$ where a horizonal electric and vertical magnetic fields are produced. The vertical gap of the dipole magnet is $680 \mathrm{~mm}$ high to accommodate the electrodes mounted inside the vacuum chamber. The electrodes are $378 \mathrm{~mm}$ high and have a $141 \mathrm{~mm}$ spacing to the grounded upper and lower walls of the vacuum chamber. The horizontal gap is $220 \mathrm{~mm}$ wide and a maximum operating high voltage of $\pm 300 \mathrm{kV}$ will be applied.

The horizontal electric field is produced by the left and right side electrodes limiting the GFR to $220 \mathrm{~mm}$. The electrode height is kept as small as possible for a high electric field by shaping the upper and lower ends of the electrodes. The homogenizing electrodes that were applied to reduce the vertical height of the electrodes of St. GEORGE [10] was not used in the SECAR design because the required triple point connections at $\pm 300 \mathrm{kV}$ on the elctrodes would have been too big to accommodate the relatively small cross sections of these electrodes causing undesireable field distortions.

The vertical magnetic field is produced by a large-gap iron-dominated dipole

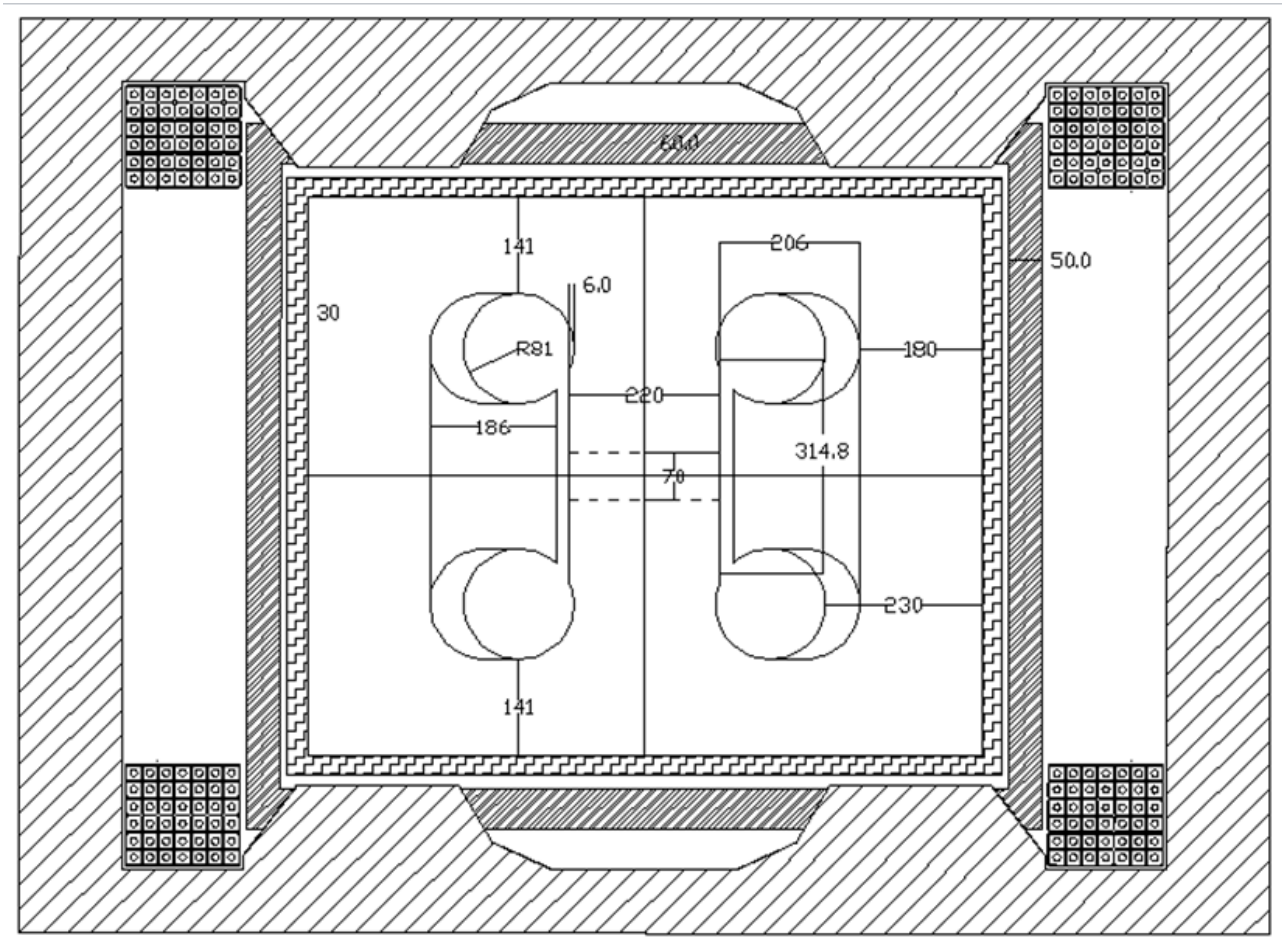

Fig. 8. Vertical cross sectional view through the center of the Wien filter. The beam travels into the page at the center of the GFR of $70 \mathrm{~mm}$ and $220 \mathrm{~mm}$ vertically and horicontally, respectively. The outer hatched region shows the magnet iron. 


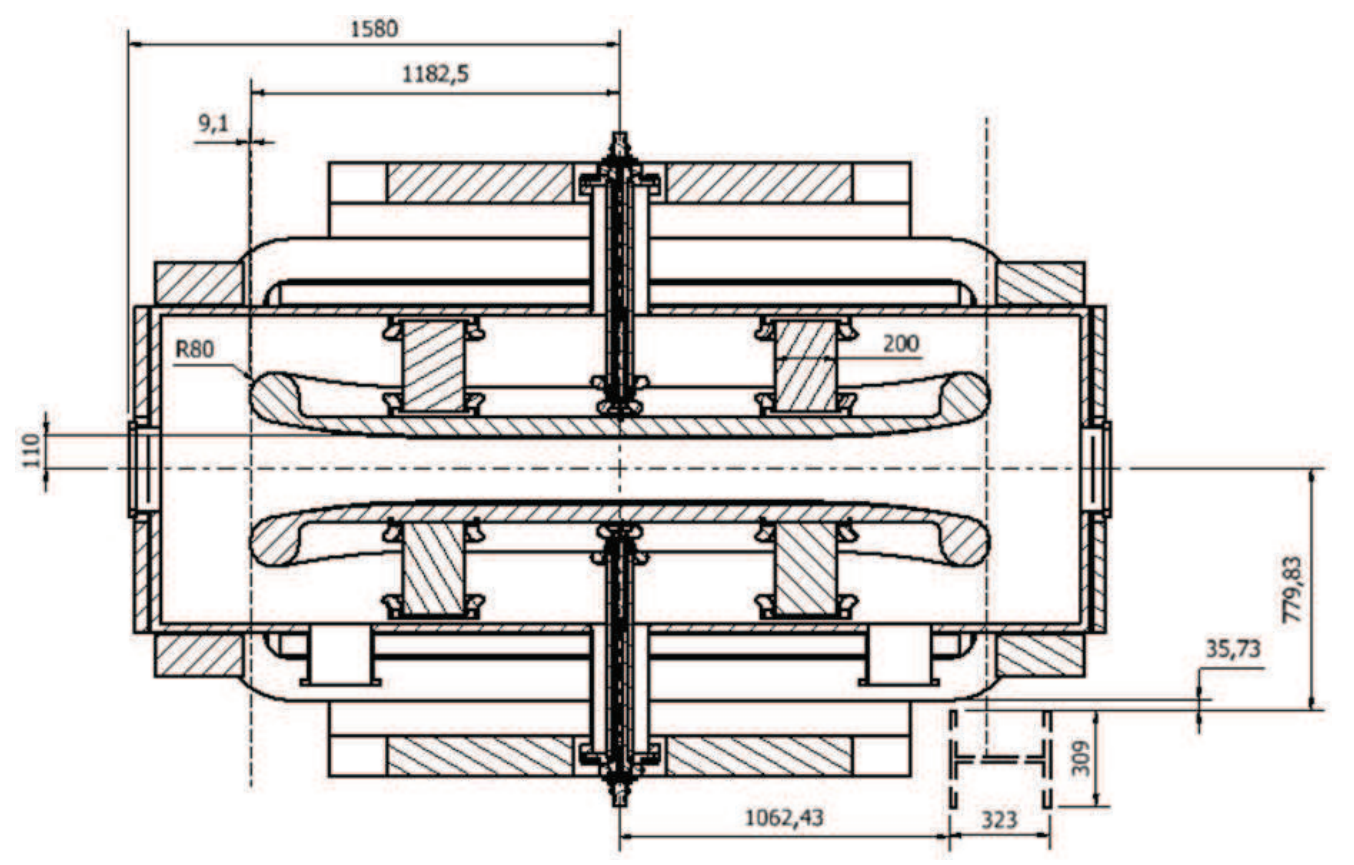

Fig. 9. A top view of the horizontal midplane of the Wien filter is shown. The electrostatic dipole is mounted inside the magnet.

magnet surrounding the vacuum chamber. The upper and lower poles are optimized to provide a constant dipole field in the GFR with high homogeneity. Also shown in the figure is one of the $50 \mathrm{~mm}$ thick adjustable field clamps mounted on both ends of the magnet to shape the fringe field and adjust the EFL of the magnet to be identical to the fixed EFL of the electric field.

Fig. 9 shows a top view of the horizontal midplane of the Wien filter. The beam enters from the left and passes between the electrodes. They are mounted from the sides on ceramic supports and are connected in the center to the high voltage power supplies by the feed-though connectors shown. The magnet coils, returns, and field clamps are shown surrounding the vacuum chamber. The effective field lengths of both the magnetic and electric dipoles are 2365 $\mathrm{mm}$.

\subsection{Wien filter condition $v=E / B$ in fringe field}

Another challenge in designing Wien filters is the difference in the spatial distribution of electric and magnetic fringe fields. Because of the large gap of the magnetic dipole that has to include the electrode height and the safe distance to ground and properties inherent to magnetic dipoles, the magnetic fringe field extends over a larger distance than the electric fringe field. In such a fringe field region, the ratio of $\mathrm{E} / \mathrm{B}$ differs from that inside the Wien filter, and this can result in a significant degradation in the performance of the 


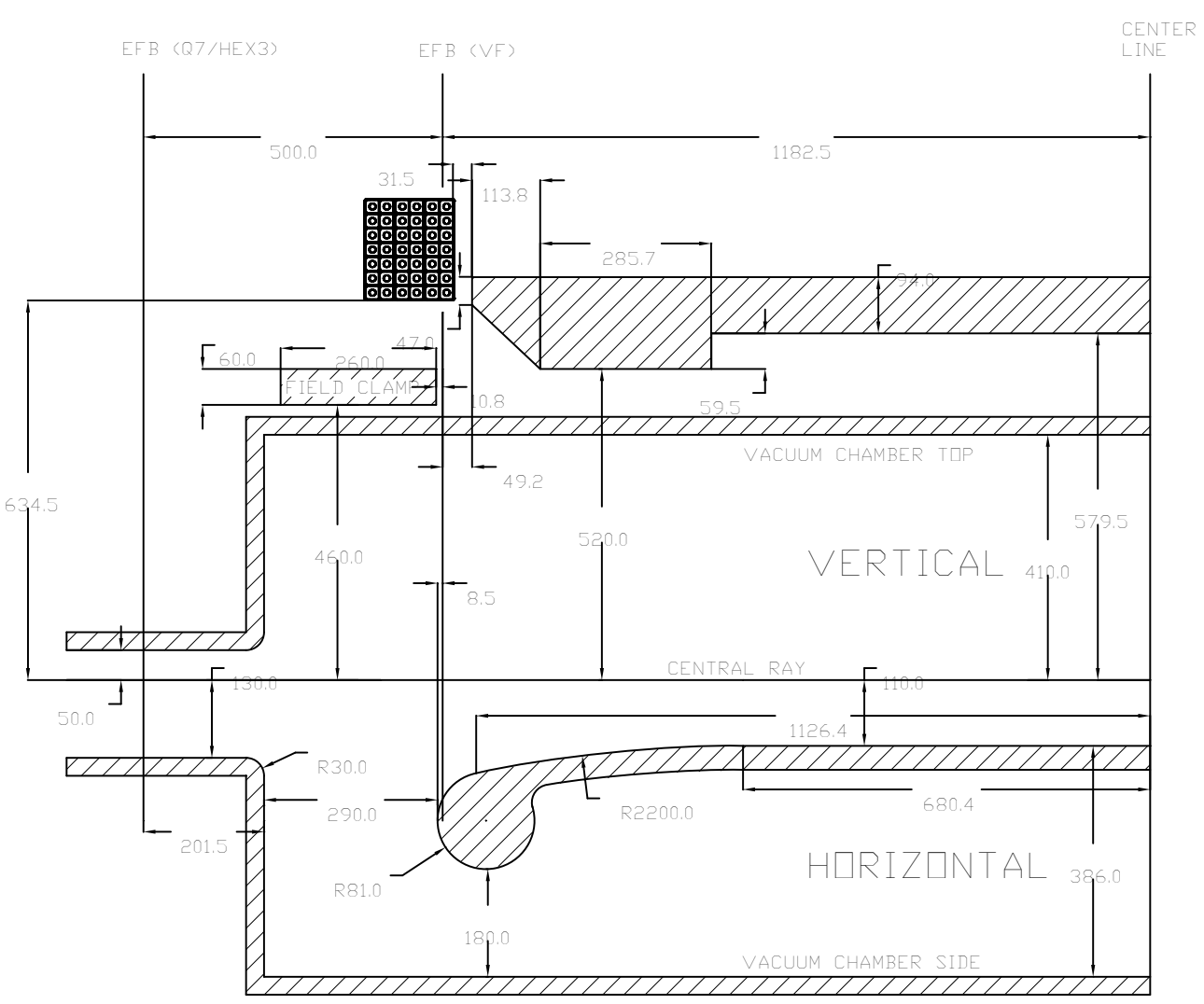

Fig. 10. A cross section view of the upper part of half the magnet is shown in the $z-y$ plane. The end pack with a chamfer of $135.1 \mathrm{~mm}$ by $127.0 \mathrm{~mm}$ and the field clamp provide the magnetic fringe field shown in Fig. 11. The lower part shows details of the electrode geometry. The resulting electric fringe field is also shown in Fig. 11.

velocity filter. Several features have been incorporated into the design of the Wien filter in an effort to minimize the distortions in the fringe field region in a similar way as discussed previously [10].

As seen in Fig. 9, the ends of the electrodes are specially shaped using 2D field calculations to ensure that the ratio $\mathrm{E} / \mathrm{B}=\mathrm{v}$ of the electric $\mathrm{E}$ and the magnetic B fields remains constant as much as possible throughout the fringe field regions to achieve the best possible velocity and mass separation. The end-packs of the magnet in the vertical mid-plane amd details of the electrode ends in the horizontal mid-plane are shown in Fig. 10. The field clamps are designed to achieve a sharp magnetic field drop off that follows the shape of the electric field in the fringe field region. This is accomplished by flaring open the electrode ends progressively as shown in Fig. 9 and 10 and by moving the grounded entrance and exit walls to a distance of $290 \mathrm{~mm}$ from the ends of the electrodes. The resulting electrode design was optimized in 2-dimensional calculations using the finite element field code OPERA [30]. The field clamps are adjustable in the direction of the central ray to be able to optimize the EFB of the magnetic field. 


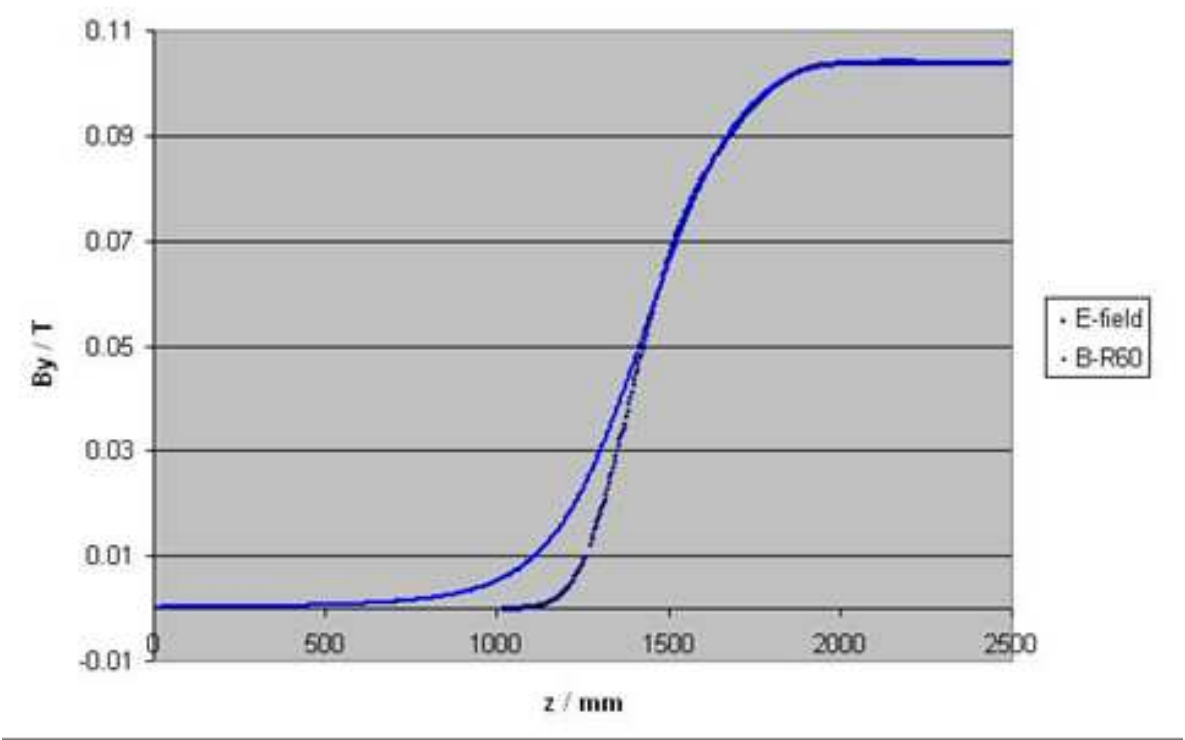

WF Magnet End pack. Ratio ElB

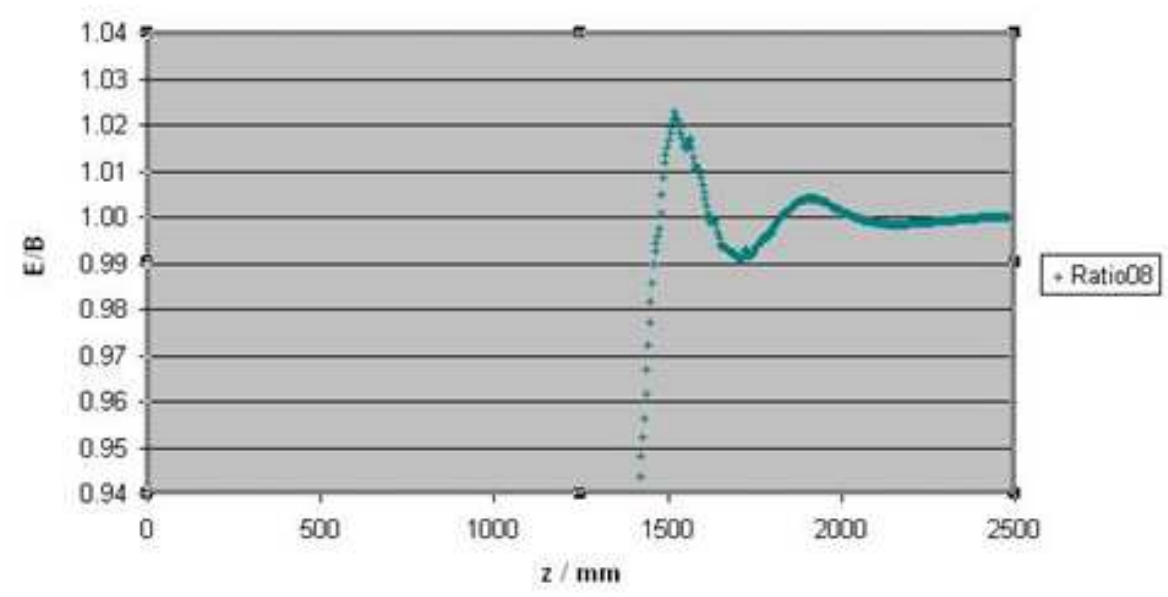

Fig. 11. Comparison of the electric and magnetic fringe fields.

A comparison of the electric and magnetic fringes fields is shown in the upper part of Fig. 11. The lower part shows the ratio E/B normalized to 1 inside the Wien filter $\mathrm{z}=2000-2500 \mathrm{~mm}$. In the fringe field region $\mathrm{z}=1440-2000 \mathrm{~mm}$ deviations of at most $2 \%$ are observed. Further outside the E/B ratio drops quickly and becomes practically 0 for $\mathrm{z} \leq 1150$. Ion-optical calculations with the Enge functions fitted to the calculated electric and magnetic fringe field showed that the mass resolution is not affected within tolerances. Subsequent 3D calculations have shown that optimizations of the electrode ends that cannot be studied in 2D calculations are important to achieve the design goals for the mass resolution. In particular soft iron mirror plates at the ends of the vacuum chamber greatly improve the $\mathrm{E} / \mathrm{B}=1$ condition compared to the $2 \mathrm{D}$ results shown in Fig. 11. 


\section{One-Wien filter option}

The presented recoil separator provides a very high mass separation of $\mathrm{m} / \Delta \mathrm{m}$ $=780$ about a factor of 2 higher larger than any presently existing recoil separator. However, the system can also be used without the second Wien filter WF2 still providing a mass separation of $\mathrm{m} / \Delta \mathrm{m}=510$ similar to other presently available systems. In this case the cleanupSection $S_{4}$ follows immediately after focal plane FP2 with identical hardware, but slightly different magnet settings to account for the different beam properties at FP2 compared to FP3. The layout and ion-optics of this option are shown in Figs. 12 and 13, respectively.

This design feature allows to build and operate the One-Wien filter version for cases where a mass separation of $\mathrm{m} / \Delta \mathrm{m}=510$ is sufficient limiting the

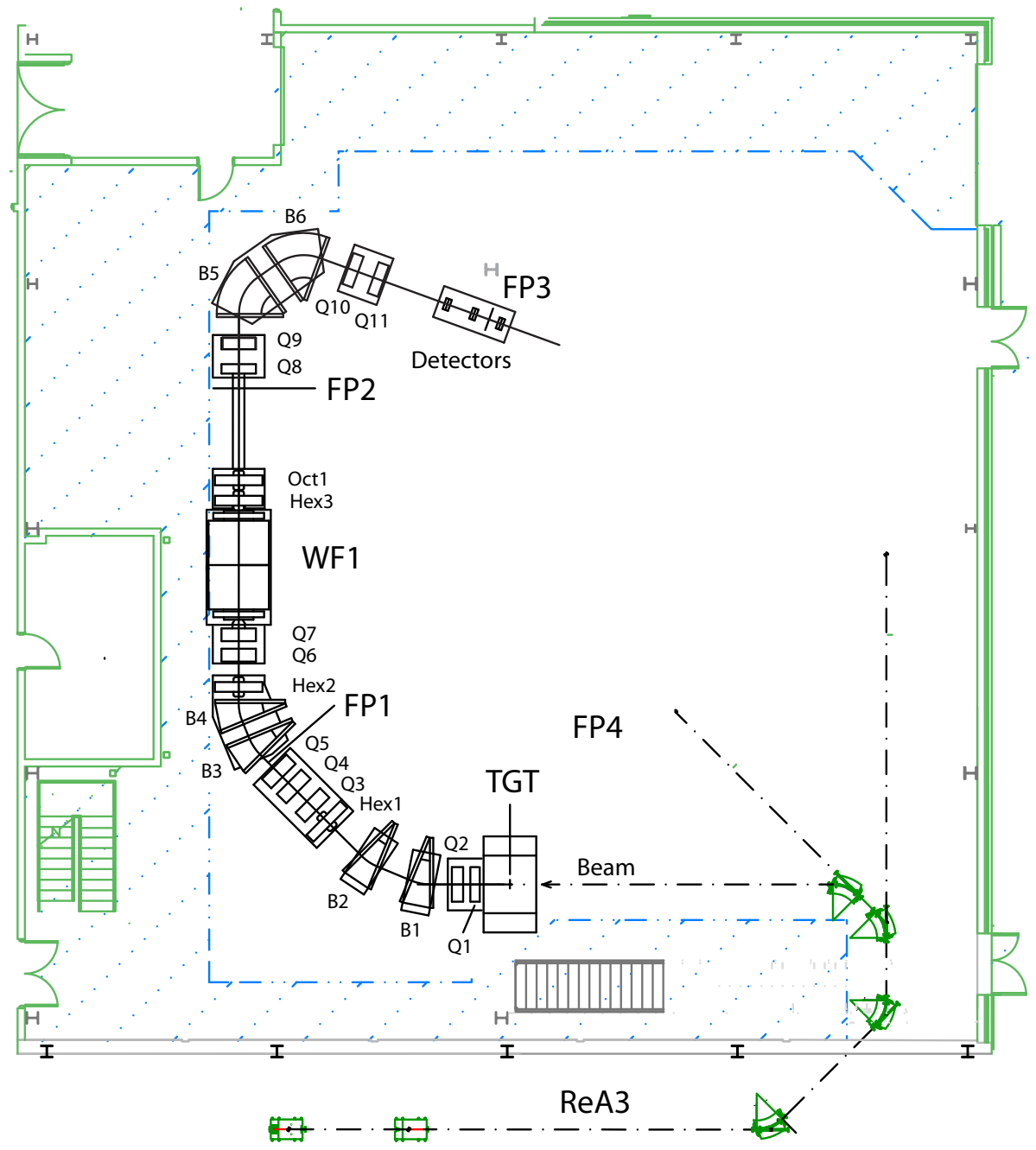

Fig. 12. SECAR floor plan with one Wien filter Section suitable to study $(\mathrm{p}, \gamma)$ and $(\alpha, \gamma)$ reactions in inverse kinematics. 


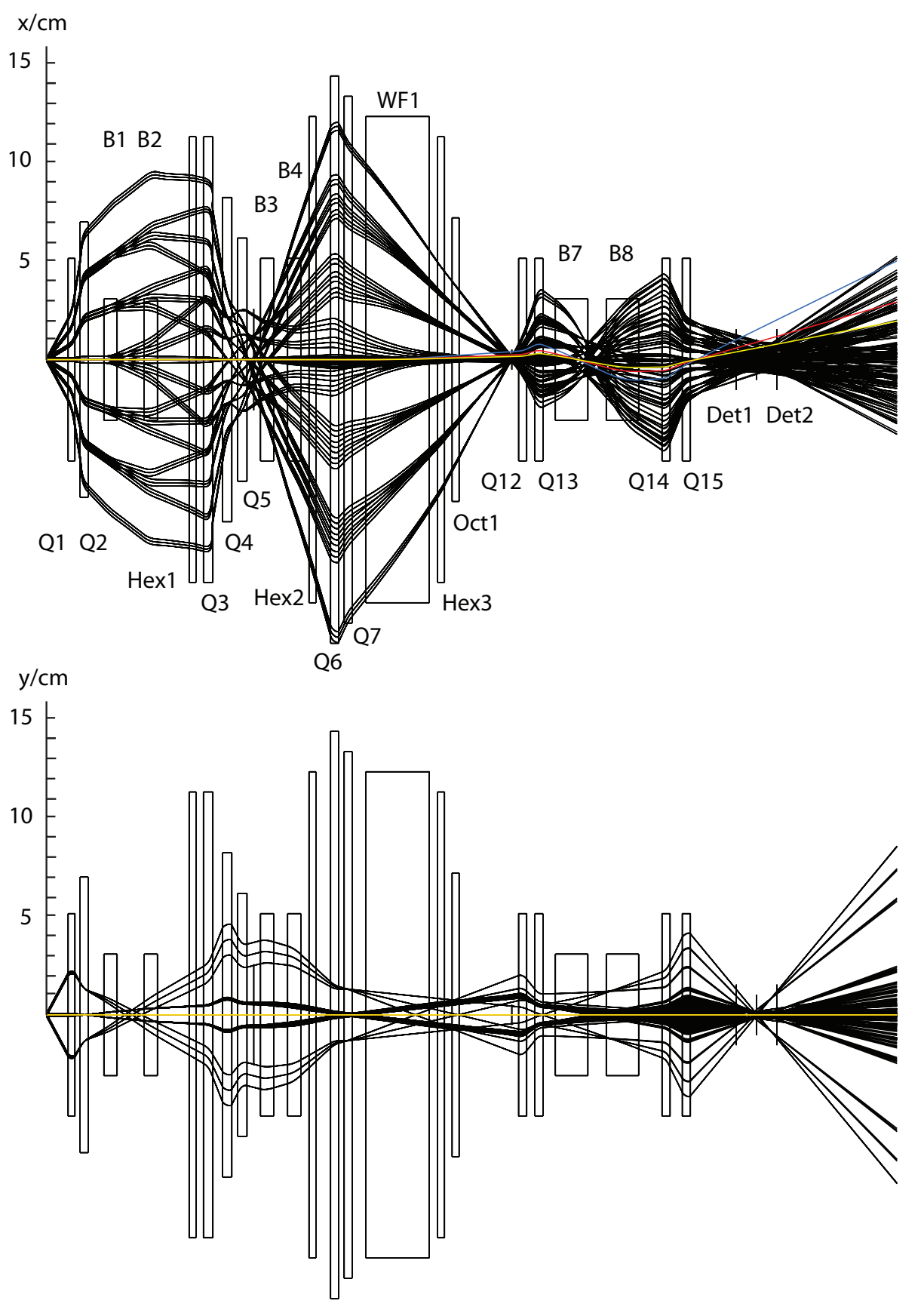

Fig. 13. SECAR ion optics for the One-Wien Filter option corrected to order 4 and calculated up to order 7. The layout is shown in Fig. 12. Shown are 189 characteristic rays in the horizontal and vertical planes in the upper and lower panel, respectively. The mass resolutions in the horizontal plane at $\mathrm{FP} 1$ is $\mathrm{m} / \Delta \mathrm{m}=510$.

target masses that can be studied to $\mathrm{A}=30-40$ below the desired maximum masses of $\mathrm{A}=65$. A later upgrade to the Two-Wien filter version to study reactions that require the higher mass separation would be needed. 


\section{Summary}

A recoil separator has been designed with the goal to measure the radiativecapture reactions $(\mathrm{p}, \gamma)$ and $(\alpha, \gamma)$ at very low energies in inverse kinematics for high intensity short-lived radioactive ion beams where $(\mathrm{p}, \gamma)$ and $(\alpha, \gamma)$ measurements in normal kinematics are not possible. The system is designed to make optimum use of the RI beam that will become available at the FRIB facility under construction at MSU. These measurements are very important to determine the stellar reaction rates used in astrophysical studies. The method of inverse kinematics requires large angular and energy acceptances of \pm 25 mrad and $\pm 3.1 \%$, respectively. Higher order ion-optical corrections are accomplished by shaping the entrance and exit field boundaries of the dipole magnets and several hexapole and octupole magnets for flexibility.

Separation of beam and reaction products is accomplished by first selecting one charge state and transmitting a beam and fusion products with almost the same momentum. Specially designed Wien filters are used for a mass selection up to $\mathrm{m} / \Delta \mathrm{m}=780$, higher than any existing recoil separator with a prodicted beam rejection of the order of up to $10^{-13}$ required for measurements of target masses up to $\mathrm{A}=65$. Further background reduction of the the order of $10^{-4}$ is accomplished in a detector system at the end of the recoil separator.

The system is also designed to allow operation with only one Wien filter WF1 useful for experiments that require a smaller mass resolution of $\mathrm{m} / \Delta \mathrm{m}=510$. This can be accomplished by omitting SectionS3.

\section{Acknowledgments}

The authors would like to express their special thanks to Georg Bollen, Thomas Glasmacher, Carl Gross, Marc Hausmann, Dave Hutcheon, I-Yang Lee, Daniela Leitner, Jerry Nolen, Chris Ruiz, and Brad Sherrill for their helpful discussions and comments. This material is based upon work supported by the U.S. Department of Energy, Office of Science, Office of Nuclear Physics, under Award Number DE-SC0014384 and Contract DE-AC02-06CH11357 and by the National Science Foundation under grant No. PHY 08-22648 and PHY-1430152 (Joint Institute for Nuclear Astrophysics and JINA-CEE). Additional funds were provided by the Michigan State University and the University of Notre Dame. 


\section{References}

[1] J. José, M. Hernanz, and C. Iliadis, Nucl. Phys. A 777 (2006) 550.

[2] H. Schatz, and K.E. Rehm, Nucl. Phys. A 777 (2006) 601.

[3] R.K. Wallace and Woosley, Ap. J. Suppl. 45 (1981) 389.

[4] R. Fitzgerald, E. Abbotoy, D.W. Bardayan, J.C. Blackmon, A.E. Champagne, A.A. Chen, U. Greife, D.W. Hill, A.N. James, R.L. Kozub, T.A. Lewis, R. Livesay, Z. Ma, S.L. Mahan, J.W. McConnell, W.T. Milner, B.H. Moazen, P.D. Parker, D.E. Pierce, M.E. Roettger, L. Sahin, D. Shapira, M.S. Smith, F. Strieder, K.B. Swartz, J.S. Thomas and D.W. Visser, Nucl. Phys. A 748 (2005) 351.

[5] D. A. Hutcheon, S. Bishop, L. Buchmann, M. L. Chatterjee, A. A. Chen, 1, J. M. D'Auria, S. Engel, D. Gigliotti, U. Greife, D. Hunter, A. Hussein, C. C. Jewett, N. Khan, M. Lamey, A. M. Laird, W. Liu, A. Olin, g, D. Ottewell, J. G. Rogers, G. Roy, H. Sprenger and C. Wrede, Nucl. Inst. Meth. Phys. Res. A498 (2003) 190.

[6] M. Couder, C. Angulo, W. Galster, J.-S. Graulich, P. Leleux, P. Lipnik, G. Tabacaru, and F. Vanderbist, Nucl. Inst. Meth Phys. Res. A 506 (2003) 26.

[7] Chris Ruiz, Uwe Greife, and Ulrike Hager, Eur. Phys. J.A. 50: 99 (2014).

[8] R. M. Kremer, C. A. Barnes, K. H. Chang, H. C. Evans, B. W. Filippone, K. H. Hahn, and L. W. Mitchell, Phys. Rev. Lett. 60 (1988) 1475.

[9] D. Rogalla, D. Schürmann, F. Strieder, M. Aliotta, N. DeCesare, A. DiLeva, C. Lubritto, A. D’Onofrio, L. Gialanella, G. Imbriani, J. Kluge, A. Ordine, V. Roca, H. Röcken, C. Rolfs, M. Romano, F. Schümann, F. Terrasi and H. P. Trautvetter, Nucl. Inst. Meth. Phys. Res. A 513 (2003) 573.

[10] M. Couder, G.P.A. Berg, J. Görres, P.J. LeBlanc, L.O. Lamm, E. Stech, M. Wiescher, and J. Hinnefeld, Nucl. Inst. Meth Phys. Res. A 587 (2007) 35.

[11] A. Aprahamian, K. Langanke, and M. Wiescher, Prog. Part. Nucl. Phys. 54 (2005) 535-613.

[12] J. P. Dufour, R. Del Moral, H. Emmermann, F. Hubert, D. Jean, C. Poinot, M. S. Pravikoff, and A. Fleury, Nucl. Inst. Meth Phys. Res. A 248 (1986) 267.

[13] G.P.A. Berg, O. C. Dermois, U. Dammalapati, P. Dendooven, M.N. Harakeh, K. Jungmann, C.J.G. Onderwater, A. Rogachevskiy, M. Sohani, E. Traykov, L. Willmann, and H.W. Wilschut, Nucl. Instr. Meth. Phys. Res. A 560 (2006) 169.

[14] A.N. James, T.P. Morrison, K.L. Ying, K.A. Connell, H.G. Price, J. Simpson, Nucl. Instr. Meth. A 267 (1988) 144. 
[15] S. Engel, D. Hutcheon, S. Bishop, L. Buchmann, J. Caggiano, K.L. Chatterjee, A.A. Chen, J. D'Auria, D. Gigliotti, U. Greife, D. Hunter, A. Hussein, C.C. Jewett, A.M. Laird, M. Lamey, W. Liu, A. Olin, D. Ottewell, J. Pearson, C. Ruiz, G. Ruprecht, M. Trinczek, C. Vockenhuber, C. Wrede, Nucl. Instr. Meth. A $553(2005) 491$.

[16] DRAGON Recoil Separator Optics. The Recoil Separator Group, TRIUMF, 18 January 1999, http://lin12.triumf.ca/text/ISAC/ISAC-pre2004/dragon/ optics_jan_18_99.pdf

[17] O. Kester, D. Bazin, C. Benatti, J. Bierwagen, G. Bollen, S. Bricker, A.C. Crawford, S. Chouhan, C. Compton, K. Davidson, J. DeLauter, M. Doleans, L. Dubbs, K. Elliott, A. Lapierre, W. Hartung, M. Johnson, S. Krause, F. Marti, J. Ottarson, G. Perdikakis, L. Popielarski, J. Popielarski, M. Portillo, R. Rencsok, D. Sanderson, S. Schwarz, N. Verhanovitz, J. Vincent, J. Wlodarczak, X. Wu, J. Yurkon, A. Zeller, Q. Zhao, A. Schempp, J. Schmidt, ReA3 - The Rare Isotope ReAccelerator at MSU Proceedings of the LINAC2010, Tsukuba, Japan, 2010, MO203, 26.

[18] S. Agostinelli, J. Allison, K. Amako, J. Apostolakis, H. Araujo, P. Arce, M. Asai, D. Axen, S. Banerjee, G. Barrand, F. Behner, L. Bellagamba, J. Boudreau, L. Broglia, A. Brunengo, H. Burkhardt, S. Chauvie, J. Chuma, R. Chytracek, G. Cooperman, G. Cosmo, P. Degtyarenko, A. Dell'Acqua, G. Depaola, D. Dietrich, R. Enami, A. Feliciello, C. Ferguson, H. Fesefeldt, G. Folger, F. Foppiano, A. Fortia, S. Garelli, S. Giani, R. Giannitrapani, D. Gibin, J.J. Gmez Cadenas, I. Gonzlez, G. Gracia Abril, G. Greeniaus, W. Greiner, V. Grichine, A. Grossheim, S. Guatelli, P. Gumplinger, R. Hamatsu, K. Hashimoto, H. Hasui, A. Heikkinen, A. Howard, V. Ivanchenko, A. Johnson, F.W. Jones, J. Kallenbach, N. Kanaya, M. Kawabata, Y. Kawabata, M. Kawaguti, S. Kelner, P. Kent, A. Kimura, T. Kodama, R. Kokoulin, M. Kossov, H. Kurashige, E. Lamanna, T. Lampn, V. Lara, V. Lefebure, F. Lei, M. Liendl, W. Lockman, F. Longo, S. Magni, M. Maire, E. Medernach, K. Minamimoto, P. Mora de Freitas, Y. Morita, K. Murakami, M. Nagamatu, R. Nartallo, P. Nieminen, T. Nishimura, K. Ohtsubo, M. Okamura, S. O’Neale, Y. Oohata, K. Paech, J. Perl, A. Pfeiffer, M.G. Pia, F. Ranjard, A. Rybin, S. Sadilov, E. Di Salvo, G. Santin, T. Sasaki, N. Savvas, Y. Sawada, S. Scherer, S. Sei, V. Sirotenko, D. Smith, N. Starkov, H. Stoecker, J. Sulkimo, M. Takahata, S. Tanaka, E. Tcherniaev, E. Safai Tehrani, M. Tropeano, P. Truscott, H. Uno, L. Urban, P. Urban, M. Verderi, A. Walkden, W. Wander, H. Weber, J.P. Wellisch, T. Wenaus, D.C. Williams, D. Wright, T. Yamada, H. Yoshida, and D. Zschiesche, Nucl. Inst. Meth. Phys. Res. A506 (2003) 250-303.

[19] M. Wang, G. Audi, A.H. Wapstra, F.G. Kondev, M. MacCormick, X. Xu, and B. Pfeiffer, Chin. Phys. C, HEP \& NP, 36(12) (2012) 1603 - 2014.

[20] K. A. Chipps, D. W. Bardayan, J. C. Blackmon, K. Y. Chae, U. Greife, R. Hatarik, R. L. Kozub, C. Matei, B. H. Moazen, C. D. Nesaraja, S. D. Pain, W. A. Peters, S. T. Pittman, J. F. Shriner, Jr., and M. S. Smith, Phys. Rev. Lett. 102 (2009) 152502. 
[21] M. Trinczek, C.C. Jewett, J.M. D'Auria, S. Bishop, L. Buchmann, A.A. Chen, S. Engel, D. Gigliotti, U. Greife, D. Hunter, A. Hussein, D. Hutcheon, J. José, A.M. Laird, M. Lamey, R. Lewis, A. Olin, D. Ottewel, P. Parker, M.M. Pavan, J.E. Pearson, J. Rogers, C. Ruiz, C. Wrede, Nucl. Phys. A 758 (2005) 729.

[22] K. A. Chipps, U. Greife, D. W. Bardayan, J. C. Blackmon, A. Kontos, L.E. Linhardt, M. Matos, S. D. Pain, S. T. Pittman, A. Sachs, H. Schatz, K. T. Schmitt, M. S. Smith, P. Thompson, Nucl. Inst. Meth. Phys. Res. A763 (2014) 553-564.

[23] J. Blackmon, private communication.

[24] Fujiwara, M.; Akimune, H.; Daito, I.; Fujimura, H.; Fujita, Y.; Hatanaka, K.; Ikegami, H.; Katayama, I.; Nagayama, K.; Matsuoka, N.; Morinobu, S.; Noro, T.; Yoshimura, M.; Sakaguchi, H.; Sakemi, Y.; Tamii, A.; Yosoi, M., Nucl. Inst. Meth. Phys. Res. A422 (1999) 484.

[25] R. Neveling, H. Fujita, F.D. Smit, T. Adachi, G.P.A. Berg, E.Z. Buthelezi, J. Carter, J.L. Conradie, M. Couder, R.W. Fearick, S.V. Förtsch, D.T. Fourie, Y. Fujita, J. Görres, K. Hatanaka, M. Jingo, A.M. Krumbholz, C.O. Kureba, J.P. Mira, S.H.T. Murray, P. von Neumann-Cosel, S. OBrien, P. Papka, I. Poltoratska, A. Richter, E. Sideras-Haddad, J.A. Swartz, A. Tamii, I.T. Usman, J.J. van Zyl, Nucl. Inst. Meth. Phys. Res. A654 (2011) 29.

[26] M. Berz, COSY Infinity, http://www.bt.pa.msu.edu/index_files/cosy.htm

[27] D. Hutcheon, L. Buchmann, A.A. Chen, J.M. DAuria, C.A. Davis, U. Greife, A. Hussein, D.F. Ottewell, C.V. Ouellet, A. Parikh, P. Parker, J. Pearson, C. Ruiz, G. Ruprecht, M. Trinczek, C. Vockenhuber, Nuc. Instr. Meth. Phys. Res. B266 (2008) 4171.

[28] L. Gialanella, A. Guglielmetti, http://www.scholarpedia.org/article/

[29] R. D. Dubois et al., Phys. Rev. A84 (2011) 027701

[30] OPERA, Software for Electromagnetic Design, Cobham Technical Services, Vector Fields Software, http://www.cobham.com/technicalservices 